Measured and Manipulated Effects of Value Similarity on Prejudice and Well-Being

\author{
Lukas J. Wolf ${ }^{1,2 *}$, Paul H. P. Hanel ${ }^{1,3}$, Gregory R. Maio ${ }^{1}$ \\ ${ }^{1}$ Department of Psychology, University of Bath, Bath, United Kingdom \\ ${ }^{2}$ School of Psychology, Cardiff University, United Kingdom \\ ${ }^{3}$ Department of Psychology, University of Essex, United Kingdom
}

Author's note. The first two authors contributed equally.

The published version can be found under: https://doi.org/10.1080/10463283.2020.1810403

* Please address correspondence to Lukas J. Wolf, Department of Psychology, University of Bath, Claverton Down, BA2 7AY Bath, United Kingdom 
We review recent research investigating the effect of shared human values on personal and social outcomes. Using more precise methods than past research, cross-sectional and experimental evidence suggests that well-being and prejudice are predicted by the extent to which people's values align (or are perceived to align) with those of other people around them. Importantly, this research shows that these effects depend on the type of values being considered and are more nuanced than prior research suggests. For example, well-being is higher among individuals who perceive their fellow citizens to share their values of power and achievement. Prejudice against immigrants is higher among individuals who value conservation more but perceive immigrants to value openness. Moreover, experimentally highlighting actual value similarities rather than mean differences improves attitudes towards outgroups. We discuss how future studies can improve our understanding of value similarity effects and their underlying mechanisms.

Keywords: Value similarity, congruence, response surface analyses, well-being, prejudice 
A number of theories and findings suggest that being or feeling dissimilar to other people has important personal and social ramifications. For instance, lower self-esteem and well-being are evident when people's personality traits are less aligned with those of people around them (Assouline \& Meir, 1987; Bleidorn et al., 2016). Similarly, seminal theories of intergroup relations suggest that prejudice against an outgroup originates from perceived dissimilarities between oneself or one's ingroup and the outgroup (e.g., belief congruence theory, Rokeach et al., 1960; selfcategorization theory, Turner et al., 1987; similarity-attraction hypothesis, Byrne, 1961; Byrne \& Wong, 1962). The role of similarity versus dissimilarity may be particularly consequential for one specific social psychological construct: human values. We present novel cross-sectional and experimental evidence examining the potential for shared values to act as a social glue that binds people together and improves well-being.

The review begins with a brief overview of values, followed by a discussion of methodological developments in studying similarity effects. We then focus on well-being and prejudice as important personal and social outcomes of similarity effects. Across six studies $(N=$ 2,271 ), we examine how actual or perceived value (dis)similarities predict these outcomes using a more precise empirical approach than past research (i.e., polynomial regressions and response surface analyses). Finally, we present five experiments $(N=618)$ testing the extent to which manipulations of value similarity reduce prejudice. Throughout this review, we discuss potential mechanisms underlying the (dis)similarity effects and suggest future directions for research on shared values.

\section{Human Values}

Human values are typically defined as abstract goals, ideals, and guiding principles in people's life (Fischer, 2017; Maio, 2016; Schwartz, 1992), which have been shown to be important predictors of well-being (e.g., Sortheix \& Schwartz, 2017) and prejudice (Davidov et al. 2008; Vecchione et al., 2012). In the present review, we focus on the the quasi-circumplex model proposed by Schwartz (1992; Schwartz \& Bilsky, 1987, 1990), which is the predominant model of 
values in psychology (Maio, 2016). According to Schwartz's model, values are ordered based on their underlying motives, with values expressing similar motives placed adjacent to each other (e.g., tradition and security) and values expressing conflicting motives placed furthest apart (e.g., tradition and stimulation; Figure 1). Its two-dimensional space contrasts self-transcendence with self-enhancement values and openness with conservation values. Self-transcendence and openness values are conceptualised as anxiety-free, whereas self-enhancement and conservation values are conceptualised as anxiety-avoidant (Schwartz et al., 2012). This two-dimensional structure has been replicated consistently across many cross-sectional and experimental studies from over 80 countries, using a range of methods (Bilsky et al., 2011; Coelho et al., 2019; Hanel, Wolfradt, et al., 2018; Maio, Pakizeh, et al., 2009; Schwartz, 1992; Schwartz et al., 2012). 


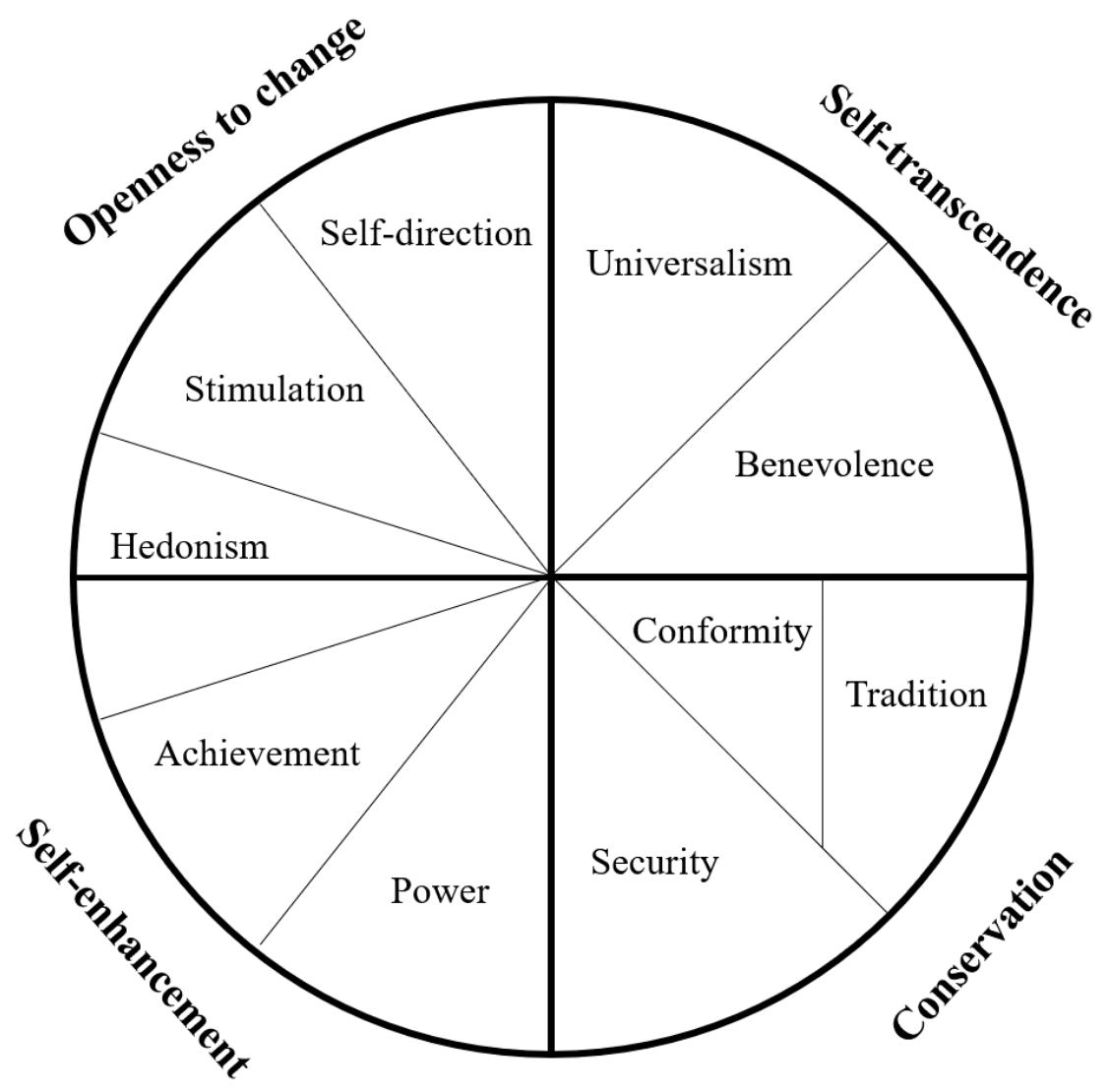

Figure 1. Schwartz' (1992) quasi-circumplex model of human values displaying four higher-order value types (bold font) and the ten value types (normal font).

Similarities in human values between individuals are an important factor in predicting and shaping a range of outcomes, including well-being (Khaptsova \& Schwartz, 2016; Musiol \& Boehnke, 2013; Sagiv \& Schwartz, 2000; Zenker et al., 2014), job satisfaction (Kristof-Brown et al., 2005; Verplanken, 2004), relationship satisfaction (Leikas et al., 2018), self-esteem (BenishWeisman et al., 2019), national pride (Du et al., 2019), and prejudice (symbolic racism theory, Kinder \& Sears, 1981; McConahay et al., 1981; integrated-threat theory, Stephan et al., 1999; see also: Allport, 1954; Merton, 1957; Parsons et al., 1951). This importance is in line with suggestions that human values are among the most fundamental psychological constructs because of their 
function as life-guiding principles that are central to individuals' self-concept (Maio, 2016; Rokeach, 1973; Schwartz, 1992; Verplanken \& Holland, 2002). Furthermore, part of the importance of these constructs derives from their abstract nature, which transcends specific situations and objects, enabling people to use values as markers of common, shared principles and ideals (cf. Boer et al., 2011). This aspect makes socially shared values particularly intriguing as potential key binding factors that may facilitate social belongingness, shared norms, and positive intergroup and interpersonal relations. Yet, this potential is relatively underexplored and there are complexities in recent theory, evidence, and methodological developments.

\section{Methodological Developments}

To test how similarities relate to outcomes such as well-being and prejudice, research in personality and social psychology has generally used (absolute) difference scores or profile correlations as indicators of (dis)similarity. Value difference scores are commonly computed between personal values and the actual or perceived values of others (e.g., a romantic partner, fellow citizens, outgroup members). These difference scores are often squared or transformed into absolute differences and then averaged across values to form an overall (absolute) difference index. This index is then correlated with variables of interest. To compute profile correlations, the value profile of one person is correlated with the (perceived) value profile of another person or group, and the resulting correlations are then correlated with variables of interest.

Despite their intuitive appeal, both analytical methods have important limitations (Edwards, 1993, 1994, 2002; Griffin et al., 1999). First, they reduce an inherently three-dimensional relationship between two sets of values and an outcome to an ambiguous two-dimensional relation between the similarity index and the outcome. Consequently, these approaches discard information that is essential to understanding similarity effects. For instance, both methods cannot identify whether similarity effects emerge predominantly at high or low levels of the component measures, and these methods miss information about whether certain types of dissimilarities are more impactful than others. To illustrate, while both approaches may show that relationship satisfaction 
is predictable from similarities between own and partner self-transcendence values, the approaches would be unable to detect that this similarity effect emerges only when own and partner values are high, but not when they are both low. Similarly, both approaches would be unable to detect that relationship satisfaction remains high when the partner has higher self-transcendence values than oneself, but that it drops when the partner has lower self-transcendence values than oneself. In addition, profile correlations discard information about the magnitude of similarities, because correlations may indicate a strong similarity effect despite large discrepancies (but similar direction and shape) between scores on the component measures.

Second, both methods conflate the contributions of the component measures with a similarity effect. For example, a dissimilarity effect is not unequivocally supported by evidence that higher (absolute) difference scores between own and perceived outgroup values predict higher prejudice. Such a finding may indicate simply that own values predict prejudice strongly, whereas perceived values are unrelated to prejudice. Third, (absolute) difference indices typically suffer from low reliability. An example provided by Edwards (2002) indicates that, when the component measures show reliabilities of .75 , are correlated at .40, and have unit variances, the resulting difference score has a reduced reliability of .58. Fourth, these methods often pose additional problems under certain circumstances. Difference scores and the relative contributions of their component measures become more ambiguous when the measures have unequal variances because measures with larger variances are weighted more heavily in difference scores. Profile correlations become more ambiguous when participants complete both component measures (e.g., own and perceived value measures), given that response biases are likely to conflate participants' correlations. Finally, and importantly for the present review, both methods are particularly problematic when researchers are interested in examining similarities in individual human values, given that values commonly consist of several types (e.g., Schwartz, 1992, 10 value types) and both approaches have typically reduced this information to one single similarity score, thus preventing the researchers from observing differences in effects among values. In short, both methods 
introduce substantial ambiguities in the analyses that are deeply problematic for interpretations of similarity effects.

Polynomial regressions and response surface analyses can be used to redress these shortcomings of difference scores and profile correlations. Although this approach has been proposed in the early 1990s (Edwards, 1993; Edwards \& Cooper, 1990), personality and social psychology has recognised its advantages only in recent years (Barranti et al., 2017; Humberg et al., 2018). A polynomial regression analysis (PRA) regresses an outcome variable (e.g., relationship satisfaction) onto the linear terms of two predictors (e.g., own and partner values), their quadratic terms, and their interaction. Response surface analyses (RSA) can plot the complex interplay between these linear terms, quadratic terms, and the interaction in three-dimensional space, allowing for visual inspection of the effects (R package 'RSA'; Schönbrodt \& Humberg, 2018). An RSA plot displays a surface of the expected values of the outcome at all possible combinations of the component measures, as derived from the polynomial regression analysis. Figure 2 shows hypothetical examples to illustrate (A) a main effect of own values, (B) a main effect of partner values, (C) quadratic effects of own and partner values, and (D) a positive interaction effect on relationship satisfaction, in the absence of other effects. For instance, the "own values" main effect in Figure 2A indicates that participants higher in "own values" (e.g., benevolence) show higher relationship satisfaction. The interaction effect in Figure 2D shows that relationship satisfaction is higher when both own and partner values are high (i.e., top back corner) and when both own and partner values are low (i.e., top front corner). In contrast, relationship satisfaction is low when own values are high and partner values are low (i.e., bottom right corner) or when own values are low and partner values are high (i.e., bottom left corner). Both predictors are centred, meaning that 0 on both the $\mathrm{X}$ and $\mathrm{Y}$ dimensions represent the midpoint of the respective scales. The lines on the ground of the plot are contour lines, with lines that are closer to each other indicating a steeper increase. The two lines shown as blue on the surface are the similarity line (running from $-2 /-2$ to $+2 /+2$ on both predictors) and the dissimilarity line (running from $-2 /+2$ to $+2 /-2$ ). 

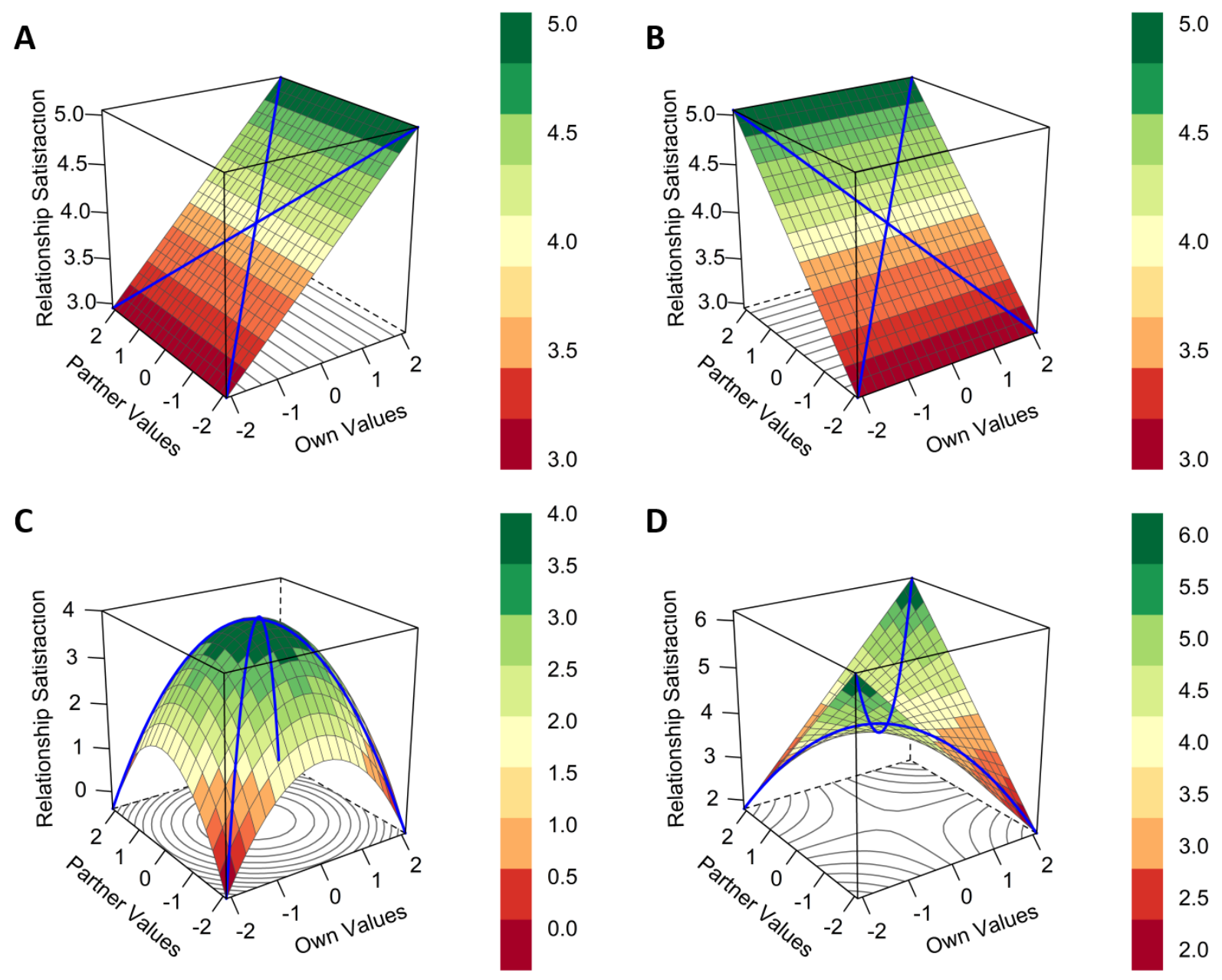

Figure 2. Hypothetical response surface analysis plots to illustrate (A) a main effect of own values,
(B) a main effect of partner values, (C) two quadratic effects of own and partner values, and
(D) a positive interaction effect on relationship satisfaction, in the absence of other effects.

Figure 3 illustrates a hypothetical example of a perfect (dis)similarity effect, which is a function of a strong interaction effect (i.e., $\beta=.50$ ) and moderate negative quadratic effects of own and partner values (i.e., $\beta=-.25$ ), in the absence of linear effects. That is, relationship satisfaction is highest (tending towards green) when "own values" and "partner values" have similar scores (e.g., both -2 or both 0 ) and lowest (tending towards red) when both predictors have dissimilar scores (e.g., -2 on one predictor and +2 on the other predictor). In other words, datapoints that are closer to the similarity line running from $-2 /-2$ to $+2 /+2$ are linked with the highest relationship 
satisfaction, whereas datapoints that are closer to the points of dissimilarity at $-2 /+2$ or $+2 /-2$ are linked with the lowest relationship satisfaction. As we will illustrate later with real datasets, this approach allows researchers to identify the location of a similarity effect (e.g., does it emerge when both own and partner values are high but not when they are low?) and differences in a dissimilarity effect (e.g., is it less detrimental for relationship satisfaction when own values are lower than partner values or the other way around?). There are excellent methodological and conceptual overviews of this approach for the interested reader (Barranti et al., 2017; Edwards, 2002; Humberg et al., 2018).
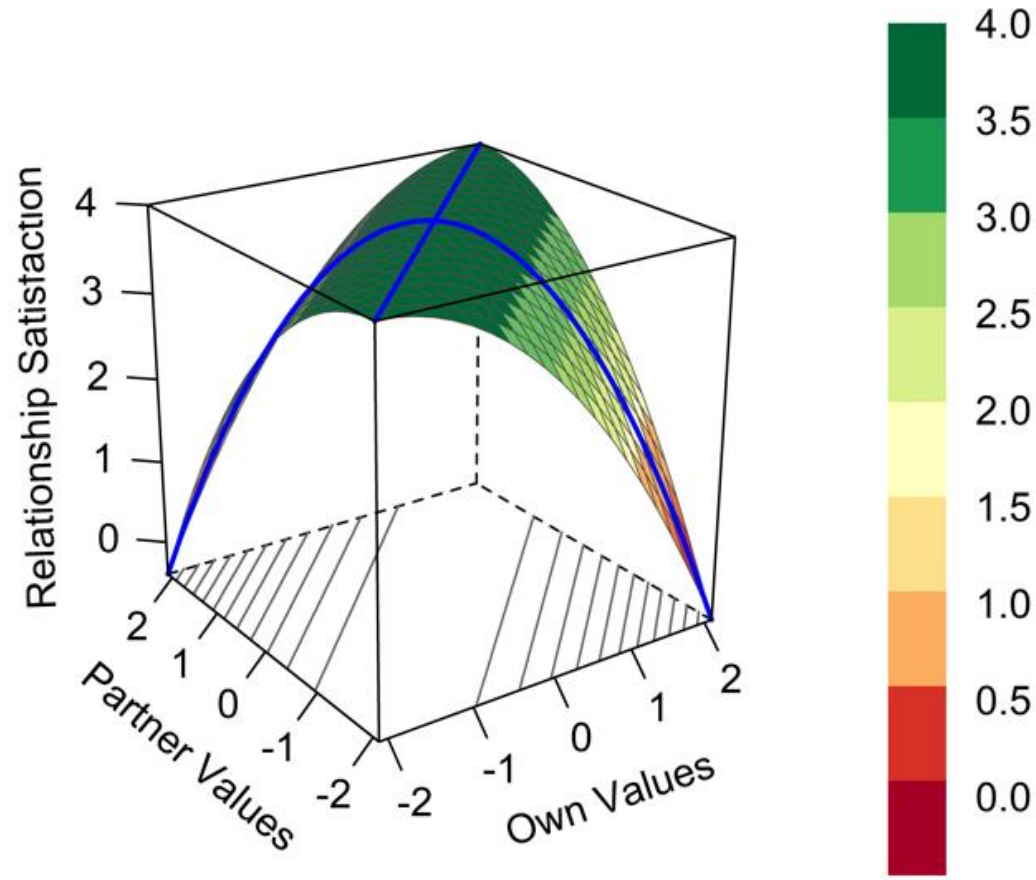

Figure 3. Hypothetical response surface analysis plot to illustrate a perfect (dis)similarity effect between own and partner values on relationship satisfaction.

Overall, then, this analytical approach redresses shortcomings of previously used methods by (1) providing essential information about similarity effects such as their location and strength, (2) examining similarity effects independently from the contributions of the component measures, (3) retaining the reliabilities of the component measures, (4) allowing for visual inspection of the 
effects in a three-dimensional space, and (5) allowing value researchers to identify similarity effects for individual value types (Barranti et al., 2017; Edwards, 2002). A combined PRA and RSA approach hence provides more statistical validity, clarity, and detail about the nature of value similarity effects. It is noteworthy here, however, that profile correlations may retain unique utility by considering congruencies between people's value hierarchies (e.g., both partners placing higher importance on benevolence as a value than on power relates to higher relationship satisfaction).

While this approach still entails the shortcomings described above, future research could explore in which ways this more holistic analysis can complement the nuanced analysis of a PRA and RSA approach.

In the remainder of this article, we describe empirical evidence applying the PRA and RSA approach to the study of value similarity effects in well-being and prejudice. We begin with research on well-being in romantic relationships because it introduces an additional important methodological and conceptual distinction within research on similarity: perceived versus actual similarity. We then proceed to discussing value similarity research on well-being in an organisational context, on well-being in a societal context, and finally, on prejudice in an intercultural context. This direction of travel follows a consideration that value similarity in research on relationships reflects a relatively narrow focus (i.e., comparing own and partner values), and this focus becomes increasingly broader for research in organisations (i.e., comparing own and organisational values), society (i.e., comparing own and fellow citizen values) and research on prejudice (i.e., comparing own and an outgroup's values). Throughout these sections, we consider potential mechanisms underlying similarity effects and provide suggestions for future directions in research on shared values.

\section{Well-Being}

Past studies have conceptualised person-environment value similarity using either a subjective or an objective approach (cf. Musiol \& Boehnke, 2013). In the subjective approach, individuals' self-rated value priorities are compared with their perceptions of the value priorities of 
a person or a group (e.g., romantic partner, family, fellow students, society). In the objective approach, individuals' self-rated value priorities are compared with the actual values of another person or aggregated self-rated value priorities of a group. These two approaches are also respectively labelled perceived and actual similarities in the interpersonal attraction literature (e.g., Montoya et al., 2008). While it has been argued that effects of perceived similarities are stronger than those of actual similarities (Condon \& Crano, 1988), a meta-analysis has found that the effects of perceived and actual similarity (of personality traits and attitudes) on interpersonal attraction depend on the level of acquaintanceship (Montoya et al., 2008). If acquaintanceship is low (i.e., with no prior interaction), the effects of both perceived and actual similarities are strong and similar in magnitude ( $r$ s $=.54$ and .49 , respectively). If the level of acquaintanceship increases, the effect sizes decrease for both types of similarities, but decrease more strongly for actual similarity. Accordingly, in established relationships (i.e., romantic partners who interacted often and across many contexts), actual similarities of personality traits and attitudes commonly do not predict attraction. These findings indicate that the importance of actual trait similarity diminishes as partners become habituated to each other, while perceptions of trait similarity begin to matter more in a relative sense. Recent research by Bleidorn et al. (2016) and Weidmann et al. (2017) suggests that a similar pattern may also emerge for well-being outcomes. That is, using the PRA and RSA approach, Bleidorn et al. showed that higher actual trait similarity with fellow citizens (i.e., low acquaintanceship) predicts higher self-esteem, whereas Weidmann et al. found negligible effects of actual trait similarity on romantic relationship satisfaction (i.e., high acquaintanceship; see also Dyrenforth et al., 2010). An interesting question then is whether the pattern obtained by Montoya et al. arises when considering how similarities in values predict well-being. In the following three sub-sections, we examine recent evidence on how value similarities within romantic relationships, organisations, and society relate to well-being outcomes. 


\section{Romantic Relationships}

Dating websites, relationship gurus, and experts have long postulated that value similarities are vital for close, romantic relationships. Drawing on interdependence theory (Kelley \& Thibaut, 1978), value similarity comes with more rewards and lower costs because partners need to negotiate and compromise less. Further, according to the goal literature, striving for a common purpose binds people together (Agnew et al., 1998; Gere et al., 2011), and people who perceive their partner as instrumental for an important goal are drawn closer to their partner (Fitzsimons \& Fishbach, 2010). Overall, past theories suggests that value similarities should predict higher relationship satisfaction, but the existing evidence on this link is limited.

We are aware of only one published study on value similarities in romantic relationships that used a PRA and RSA approach. In this study, Leikas et al. (2018) used the Portrait Values Questionnaire (PVQ; Schwartz et al., 2012) to examine actual similarities in 312 Finnish heterosexual couples. Only actual similarities in self-direction values predicted relationship satisfaction in men and women, with satisfaction being highest if partners valued self-direction equally in a medium range. ${ }^{1}$ This finding suggests that couples are dysfunctional when one partner values freedom, independence, and curiosity (i.e., self-direction values) significantly more than the other (cf. work on attachment insecurity by Overall et al., 2015). In other words, and as discussed above, such dissimilarities in self-direction values may reflect conflicting life goals, requiring negotiation and compromise. Further, both partners strongly valuing their self-direction may conflict with the idea of being in a co-dependent relationship, whereas both partners placing very low importance on their freedom may potentially result in an unhealthy co-dependence (e.g., Aryamanesh et al., 2012). However, equally valuing self-direction at a moderate level may strike a balance, resulting in the highest level of relationship satisfaction.

\footnotetext{
${ }^{1}$ Benevolence and conformity values showed weak main effects on relationship satisfaction. Higher satisfaction was reported when own conformity values were higher, when partner conformity values were higher, when men's own benevolence values were higher and when women's partner's benevolence values were higher. These main effects are comparable to the effect size found for the similarity effect in self-direction values.
} 
Leikas et al. (2018) did not find evidence of similarity effects in other values. The finding that actual value similarities do not strongly predict relationship satisfaction is in line with the aforementioned literature describing attitude and trait similarity effects on attraction and well-being (Montoya et al., 2008; Weidmann et al., 2017): Effects of actual similarities are negligible if people know each other well. Values might be too abstract to matter for daily interactions and decisions that are common in close, romantic relationships. For example, although both partners might value stimulation, one might find rock climbining more stimulating and the other watersports. In other words, partners who show high value similarity may nevertheless be incompatible in their value instantiations (e.g., the behaviours they associate with the values), potentially resulting in low relationships satisfaction. Accordingly, we suggested in previous work that value instantiations might be an important moderator of value similarity effects (Hanel et al., 2017; Maio, 2010; Maio, Hahn, et al., 2009): Similarities in certain values should only predict relationship satisfaction if both partners understand or instantiate the values in a similar way. Future work may benefit from exploring this possibility, potentially in a longitudinal study which additionally considers the role of relationship duration. For example, at the beginning of a relationship, similarities in abstract values might be more important (e.g., do both partners value achievement to a similar extent?). However, after a relationship has lasted for some time, similarities in more specific goals and behaviours (i.e., instantiations) might become more relevant for the daily interactions and decisions common in established relationships.

Another interesting avenue for future research is to test whether stronger value similarity effects emerge when perceived partner values are measured. That is, a direct comparison between actual and perceived value similarity is useful to test whether the pattern identified in Montoya et al.'s (2008) meta-analysis replicates for value similarity using the PRA and RSA approach. Such a finding that perceived, but not actual, value similarities predict relationship satisfaction may indicate that perceptions of similarity are illusory and biased (Murray et al., 2002), consistent with 
evidence showing marked differences between others' actual values and how people tend to perceive them (Bernard et al., 2006; Fischer, 2006; Hanel, Wolfradt, et al., 2018).

There is already abundant research in the relationship literature showing that perceptions and evaluations of close others are often biased (e.g., Gagné \& Lydon, 2004; Lee et al., 2009), typically in a positive direction (e.g., halo effect; Thorndike, 1920). These biases are also associated with personality characteristics. For example, highly avoidant individuals are more likely to overestimate the intensity of their partner's negative emotion (Overall et al., 2015), and individuals who score higher on depression underestimate their partner's commitment more (Overall \& Hammond, 2013). Similar biases may also emerge for values. Highly avoidant individuals might overestimate partner values that they perceive as less important (e.g., hedonism), while underestimating partner values they perceived as more important (e.g., helpfulness). Avoidant individuals may hence be more likely to perceive their partner to be dissimilar, which may contribute to lower relationship satisfaction. Future research may benefit from considering such potential moderators of perceived value similarity effects on relationship satisfaction. Moreover, to better understand the source and nature of biases present in perceived value similarities, future longitudinal work could consider the application of prior methods examining partners' accuracy in tracking each other's emotions over time (e.g., Overall et al., 2019). For instance, researchers could study how and when the perceived values of a partner can be predicted from the partner's actual values across a longer period of time.

\section{Organisations}

Much research on the link between value similarities and well-being originates in organisational research (Edwards, 2008). Theories of person-organisation fit (e.g., Holland, 1997; Joyce \& Slocum, 1984) have argued that a "shared understanding of how individuals in general impute meaning to environments" (James, 1982, p. 220) can have beneficial outcomes for job satisfaction (see also Kristof-Brown et al., 2005). Based on the notion that values allow people to make sense of their organisational environments (Chatman, 1989), research in organisational 
psychology has often concentrated on value similarities and how they predict job satisfaction. Such value similarities are often calculated between employees' values and the perceived or actual values of the supervisor, team, job, or organisation. Although past research has been limited by the employed analytical methods (e.g., use of profile correlations or difference scores as indices of value similarity), the existing evidence on this topic using the PRA and RSA approach and Schwartz's values supports the notion that actual value similarities in an organisational context do predict higher job satisfaction. For instance, Byza et al. (2019) assessed Schwartz's values using the PVQ in 116 employee-supervisor dyads and measured employees' job satisfaction and affective commitment. Actual value similarities generally predicted higher job satisfaction and affective commitment in employees across all four higher-order value types (i.e., self-transcendence, selfenhancement, openness, conservation); a pattern that was most pronounced for self-transcendence values on both outcomes and for openness values on job satisfaction. The findings also showed that these similarity effects emerge more strongly at more extreme levels of the underlying values, that is, when both employee and supervisor agree a particular value is very important or very unimportant (rather than moderately important). ${ }^{2}$

These findings indicate that actual value similarities predict higher well-being in the workplace. The findings, together with effects on relationship satisfaction discussed above, are in line with the meta-analytic pattern obtained by Montoya et al. (2008): At higher levels of acquaintanceship (e.g., in romantic relationships), the effects of actual similarity are weak, whereas at relatively lower levels of acquaintanceship (e.g., workplace) effects emerge more strongly. It is worth noting however, that the evidence reviewed here is scarce and that more work is needed using the PRA and RSA approach to draw clearer conclusions about the pattern of findings. Some similarity effects may also heavily depend on context. For instance, it is conceivable that

\footnotetext{
${ }^{2}$ Main effects of employee values showed that higher self-enhancement values and higher openness values predicted lower affective commitment and job satisfaction. Higher supervisor conservation values predicted lower affective commitment. These main effects are comparable to the effect sizes found for the similarity effects. All other main effects were non-significant.
} 
similarities in self-enhancement values (i.e., power, achievement) in the workplace predict higher job satisfaction, but only when sufficient resources are available to satisfy individuals' drive for status, wealth, and success. When resources are constrained, zero-sum competitions among employees may result in stress and reduced well-being. In addition to the availability of resources, the particular workplace culture and organisation (e.g., stable vs. unstable hierarchies; Knight \& Mehta, 2017) could also play a role. Future research may wish to explore under which circumstances value similarities have positive or negative effects in the workplace, taking into account the role of (perceived) goal facilitation or conflict.

Future work could also consider that similarities in concrete value instantiations may be important in the workplace. For instance, for employees it may not only matter if their supervisor shares the importance they attribute to achievement values, but also whether they understand such values in a similar way (e.g., low absenteeism, collegiality, efficiency). It may also be beneficial to to study potential mediators (e.g., goal conflict; feeling validated) and moderators (e.g., job security, performance, value instantiations) to better understand the underlying process. Another complexity in detecting patterns across studies derives from Kristof-Brown et al.'s (2005) meta-analysis, which showed that similarity effects differ substantially depending on the particular reference target within the organisation (e.g., supervisor, team, organisation, job). Researchers may benefit from directly comparing value similarity effects among different reference targets to identify which values play a stronger role in which contexts.

\section{Society}

The previous subsections on well-being suggested that sharing values with a romantic partner is only weakly linked with relationship satisfaction, whereas sharing values with a supervisor in the workplace generally predicts higher job satisfaction across values. This subsection concerns comparisons of values in a broader sense: To what extent is sharing (or perceiving to share) the values of fellow citizens linked with higher well-being? A host of researchers have either explicitly or implicitly adapted the reasoning on similarity effects in organisational research to form 
the prediction that sharing values with people from the same region or country should also result in higher general well-being. However, past research linking value similarities to general well-being has relied on difference scores and profile correlations. In one unpublished study from our lab which we describe below, we examined this association using the PRA and RSA approach, allowing us to test whether perceived similarity effects on well-being depend on which values are considered.

Do people report higher well-being when they perceive their fellow citizens to share their values? To answer this question, we collaborated with a non-profit organisation, the Common Cause Foundation (valuesandframes.org), and recruited a representative sample of 1181 citizens of Greater Manchester, England. Participants took part in an online survey that entailed completing the 21-item PVQ twice (Schwartz et al., 2001): once to indicate their own values and a second time to indicate their perceived values of fellow citizens of Greater Manchester. Participants also answered the question: “Overall, how satisfied are you with your life nowadays?”.

We discuss the analyses here in detail for illustration of the combined PRA and RSA approach, and because the findings are not published elsewhere. We focused on the four higherorder value types. The RSA plots are shown in Figure 4 for (A) self-transcendence values, (B) selfenhancement values, (C) openness values, and (D) conservation values. Visual inspection of the RSA plots suggests similarity effects for self-transcendence, self-enhancement, and openness values, but no effects for conservation values. That is, when participants perceived fellow Manchester citizens to share their self-transcendence, self-enhancement, and openness values, they reported higher life satisfaction, whereas this effect did not emerge for conservation values. 
A

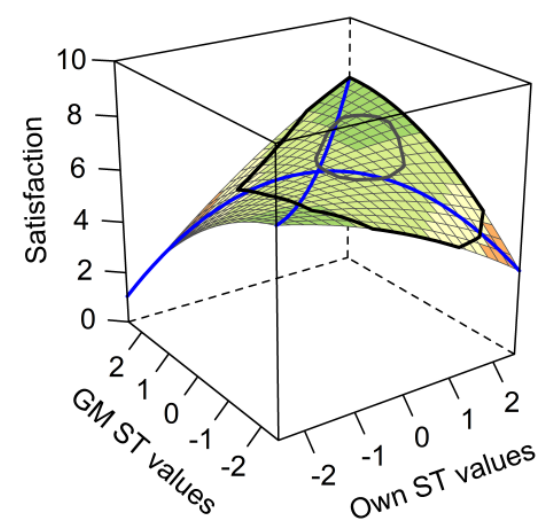

C

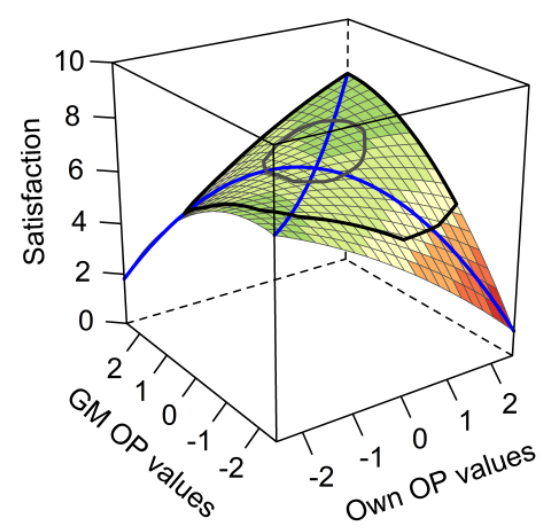

B

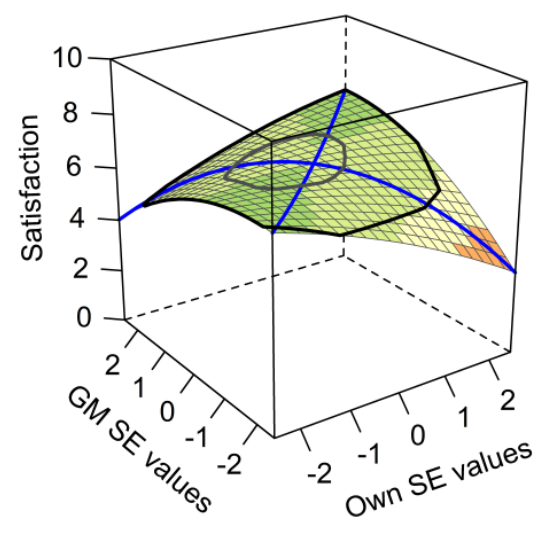

D

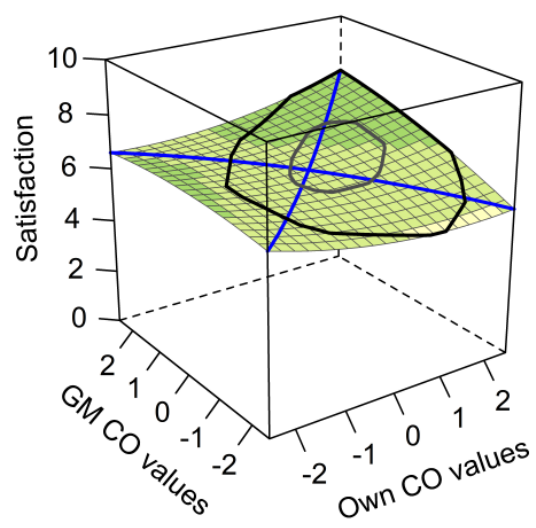

Figure 4. Response surface analysis plots to illustrate effects between own values and the perceived values of fellow citizens from Greater Manchester (GM) for (A) self-transcendence (ST), (B) selfenhancement (SE), (C) openness (OP), (D) and conservation values (CO). Plots include bagplots with $50 \%$ of the data within the inner grey line and $50 \%$ of the data within the outer black line.

The PRA coefficients in Table 1 support this initial visual inspection. For selftranscendence, self-enhancement, and openness values, the interaction terms are significant, while the quadratic terms are more moderate and tend to point in a negative direction. All main effects are non-significant. This pattern thus resembles the hypothetical example in Figure 3 of a perfect similarity effect. In contrast, conservation values show only a weak main effect of perceived values, indicating that perceiving others to value conservation more predicts higher life satisfaction. There are no indications of a similarity effect in conservation values on life satisfaction. These 
findings can be further corroborated using six RSA coefficients, $a_{1-4}, p_{10}$, and $p_{11}$. These coefficients allow researchers to test the extent to which the data conforms to a perfect similarity effect in further detail. Humberg et al. (2018) suggest testing four conditions to establish a similarity effect in a broad sense, and two additional conditions to establish a similarity effect in a strict sense.

Table 1

Polynomial regression analyses coefficients on life satisfaction.

\begin{tabular}{lcccc}
\hline & ST & SE & OP & CO \\
\hline Own values & 0.09 & -0.04 & -0.01 & 0.01 \\
Perceived values & -0.09 & 0.04 & 0.06 & $0.10^{*}$ \\
Interaction & $0.31^{* * *}$ & $0.15^{* * *}$ & $0.26^{* * *}$ & 0.05 \\
Own & & -0.07 & -0.05 & 0.05 \\
Perceived $^{2}$ & $-0.11^{* *}$ & $-0.09^{*}$ & $-0.16^{* * *}$ & -0.02 \\
\hline
\end{tabular}

Note. ST: Self-transcendence, SE: Self-enhancement, OP: Openness, CO: Conservation. All values are standardized betas. * significant at .05 level, ** significant at .01 level, $* * *$ significant at .001 level.

First, to establish whether the surface has the expected "saddle" shape as shown in Figure 3, researchers can inspect $a_{4}$, which is computed by adding the two quadratic term coefficients and subtracting the interaction term coefficient. Higher negative $a_{4}$ values hence indicate stronger positive interaction terms and stronger negative quadratic terms, pointing to a similarity effect. As can be seen in Table 2, $a_{4}$ is negative and significant for self-transcendence, self-enhancement, and openness values, but not for conservation values, supporting the initial conclusions drawn from the RSA plots and PRA coefficients. The following two conditions test the intercept and slope of the first principal axis, which is the "ridge" of the RSA surface. That is, the confidence interval of the 
intercept of the first principal axis, $p_{10}$, needs to include 0 , and the confidence interval of its slope, $p_{11}$, needs to include 1 . When the confidence interval of $p_{10}$ includes 0 and the confidence interval of $p_{11}$ includes 1 , the "ridge" of the surface can be said to run along the similarity line. When the confidence interval of $p_{10}$ does not include 0 , the ridge runs parallel to the similarity line to the left or right side, whereas when the confidence interval of $p_{11}$ does not include 1 , the ridge is turned towards the left or right side. As can be seen in Table 2, for all four value types, the confidence intervals of $p_{10}$ include 0 and the confidence intervals of $p_{11}$ include 1 , indicating that the ridge of the surface does not deviate from the similarity line. For the fourth condition, $a_{3}$, which in this context is computed by subtracting the coefficient for perceived values from own values, needs to be non-significant. A significant $a_{3}$ would indicate that the shape is tilted towards one or the other side, suggesting that the ridge of the shape does not reflect maximum life satisfaction. This fourth condition is met, with $a_{3}$ being non-significant in all four RSA analyses. Hence, the RSA coefficients confirm similarity effects for self-transcendence, self-enhancement, and openness values. 
Table 2

Response surface analyses coefficients on life satisfaction.

\begin{tabular}{|c|c|c|c|c|}
\hline & ST & SE & OP & $\mathrm{CO}$ \\
\hline \multirow[t]{2}{*}{$a_{1}$} & 0.05 & -0.01 & 0.15 & $0.27 *$ \\
\hline & {$[-0.32,0.41]$} & {$[-0.21,0.19]$} & {$[-0.13,0.42]$} & {$[0.02,0.52]$} \\
\hline \multirow[t]{2}{*}{$a_{2}$} & 0.17 & 0.07 & 0.14 & 0.12 \\
\hline & {$[-0.02,0.37]$} & {$[-0.12,0.26]$} & {$[-0.06,0.34]$} & {$[-0.06,0.31]$} \\
\hline \multirow[t]{2}{*}{$a_{3}$} & 0.44 & -0.18 & -0.17 & -0.22 \\
\hline & {$[-0.10,0.98]$} & {$[-0.47,0.11]$} & {$[-0.52,0.18]$} & {$[-0.61,0.17]$} \\
\hline \multirow[t]{2}{*}{$a_{4}$} & $-0.68 * * *$ & $-0.49 * * *$ & $-0.84 * * *$ & -0.06 \\
\hline & {$[-1.05,-0.32]$} & {$[-0.75,-0.23]$} & {$[-1.17,-0.50]$} & {$[-0.41,0.29]$} \\
\hline \multirow[t]{2}{*}{$p_{10}$} & -0.58 & 0.31 & 0.19 & 2.05 \\
\hline & {$[-1.24,0.09]$} & {$[-0.14,0.76]$} & {$[-1.24,0.09]$} & {$[-5.06,9.15]$} \\
\hline \multirow[t]{2}{*}{$p_{11}$} & 0.83 & 0.73 & 0.73 & 0.35 \\
\hline & {$[0.37,1.28]$} & {$[0.28,1.19]$} & {$[0.39,1.07]$} & {$[-0.44,1.14]$} \\
\hline
\end{tabular}

Note. ST: Self-transcendence, SE: Self-enhancement, OP: Openness, CO: Conservation. Response surface analyses coefficients shown with $95 \%$ confidence interval in brackets. * significant at .05 level, ** significant at .01 level, *** significant at .001 level.

The final fifth and sixth condition to establish similarity effects in a strict sense are tested by considering the RSA coefficients $a_{1}$ and $a_{2}$. If $a_{1}$ is significant, the saddle shape is inclined or declined (e.g., higher life satisfaction at $+2 /+2$ than at $-2 /-2$ ). If $a_{2}$ is significant, values increase or decrease at extreme levels of the predictors (e.g., simultaneously higher life satisfaction at $+2 /+2$ and at -2/-2). In our dataset, both RSA coefficients are non-significant for all three value types, suggesting that the surface is not additionally influenced by the linear terms. This study hence establishes similarity effects in a strict sense for self-transcendence, self-enhancement, and 
openness values. In other words, participants who perceived their fellow citizens to have similar self-transcendence, self-enhancement, and openness values as themselves reported higher life satisfaction than those who perceived their fellow citizens to have dissimilar values. We can also conclude that these effects emerged consistently across high and low levels of the predictors.

Finally, the significant interaction terms can be followed-up with simple slopes analyses, further illustrating the richness of this analytical approach. These follow-up analyses examine whether the effects of own values on life satisfaction emerge more strongly at one end of perceived values (e.g., at +2) than at the other end (e.g., at -2). In other words, we test whether the slope of expected values towards the "back wall" in Figure 4 is significant (effect of own values at high levels of perceived values) and whether the slope of expected values towards the "front wall" is significant (effect of own values at low levels of perceived values). These analyses indicate that participants with higher self-transcendence values reported higher life satisfaction when they perceived fellow citizens to share these values $(+1 S D=1.24)^{3}$, whereas this effect was nonsignificant when fellow citizens were perceived to be lower in self-transcendence values $(-1 S D=-$ $0.72)^{4}$. Similarly, participants who valued openness values more reported higher life satisfaction when they perceived fellow citizens to share these values $(+1 S D=1.36)^{5}$, but this effect was nonsignificant when fellow citizens were perceived to be lower in openness values $(-1 S D=-0.36)^{6}$. In contrast, participants who valued self-enhancement more reported higher life satisfaction at high levels of perceived values $(+1 S D=1.12)^{7}$, and participants who valued self-enhancement values less reported higher life satisfaction at low levels of perceived values $(-1 S D=-0.71)^{8}$. Hence, although the previous RSA coefficients indicate that the surface of expected values conform to a similarity effect that is evenly balanced, these follow-up analyses suggest that the similarity effects for self-

\footnotetext{
${ }^{3}$ Significant at $b=0.77, S E=0.22, p<.001$.

${ }^{4}$ Non-significant at $b=0-.07, S E=0.16, p=.67$

${ }^{5}$ Significant at $b=0.65, S E=0.12, p<.001$.

${ }^{6}$ Non-significant at $b=-0.19, S E=0.11, p=.089$.

${ }^{7}$ Significant at $b=0.22, S E=0.08, p=.006$.

${ }^{8}$ Significant at $b=-0.29, S E=0.12, p=.013$.
} 
transcendence and openness values are particularly evident when citizens are perceived to be higher in these values. In contrast, the similarity effects for self-enhancement values were equally balanced at both high and low levels of perceived self-enhancement values. ${ }^{9}$

It is important to note that the reported study used a large, representative sample and detailed analyses. Overall, this study showed that citizens of Greater Manchester report higher life satisfaction when fellow citizens are perceived to share their self-transcendence and openness values, but only if the citizens attach high importance to these values. Citizens also report higher life satisfaction when fellow citizens are perceived to share their self-enhancement values, irrespective of whether citizens themselves attach high or low importance to self-enhancement values. In contrast, perceived similarities in conservation values are inconsequential for citizens' life satisfaction.

To interpret these findings, we refer back to Schwartz's (1992) theoretical assumptions about the motives underlying the values. Based on these assumptions, the finding that people higher in self-transcendence values are happier when they perceive themselves to be around likeminded others suggests that striving towards common self-transcendence goals such as social cohesion and harmony is beneficial for one's well-being. Similarly, people higher in openness values who attach high importance to such principles as curiosity, freedom, and creativity may be happier around like-minded others because they allow them to satisfy their thirst for new experiences. When these self-transcendent and openness values are of lower importance, however, similarity matters less, perhaps indicating that attaching lower importance to these values is a weaker social binding factor because it implies indifference for relatively positive societal principles (e.g., social cohesion or curiosity).

\footnotetext{
${ }^{9}$ In addition to life satisfaction, we assessed several other outcomes: participants' civic engagement attitudes (e.g., "I believe that it is important to volunteer"; Doolittle \& Faul, 2013), civic participation (e.g., voting in local elections, attending demonstrations), views of social and environmental issues on poverty, climate change, and housing in Greater Manchester (e.g., "Everyone in Greater Manchester should be able to live in a home that they can afford"), views on devolution (e.g., "Do you think that having a mayor of Greater Manchester will be a good thing or a bad thing for Greater Manchester?"), likelihood to vote in the Greater Manchester mayoral election, and voting in the EU referendum (i.e., remain vs leave). None of these outcomes showed effects of value similarity.
} 
The similarity effects for self-enhancement values at high and low levels of personal importance for these values indicate that (a) people who value power and achievement may find it more rewarding when they perceive themselves to be around others who acknowledge the importance of their achievement and power and that (b) people who do not greatly value power and achievement values may find it more rewarding when they perceive themselves to be around others who also do not greatly value power and achievement. It may be the case that the role of competition in self-enhancement values is a contributing factor to this contrasting pattern. People who value power and achievement are more competitive (Hanel, Litzellachner, \& Maio, 2018) and the need to be competitive is more likely to be fulfilled if others around them are also competitive. In other words, being around people who are perceived to value self-enhancement may give their own self-enhancement values purpose and meaning, resulting in higher well-being (cf. Sagiv \& Schwartz, 2000). Conversely, people who do not cherish these values are less competitive (Hanel, Litzellachner, \& Maio, 2018) and may feel more comfortable around similarly non-competitive individuals.

Nevertheless, it is surprising that individuals who believe that their fellow citizens share their conservation values do not report higher life satisfaction than those who perceive dissimilarity in these values, particularly given that values such as conformity and security require collective action to be effective. It is possible that individuals higher in conservation values do not associate sharing their values with feelings of satisfaction but rather with feelings of relief, because conservation values involve anxiety-avoidant motivations, and a focus on avoiding outcomes has been linked with feelings ranging from anxiousness to relief (self-regulation theory; Higgins et al., 1997). In other words, for individuals higher in conservation values, perceived similarity might be related to an affective aspect of well-being that is different from life satisfaction.

It is also interesting that we find positive similarity effects for self-transcendence and selfenhancement values. At a first glance, this finding may seem to contradict Schwartz's circumplex model of values. This model indicates that opposing value types should be associated with external 
variables (e.g., attitudes, behaviour) in opposite directions (e.g., self-transcendence positively and self-enhancement negatively predicts the external variable) because they subsume conflicting motivational processes (Schwartz et al., 2012). However, similarity effects in values intrinsically involve different motivational processes than linear effects of values. Whereas values directly express particular motivational content and are typically considered positive in nature (Hitlin \& Piliavin, 2004), perceiving other people to share these values putatively engages additional motives, including a sense of common purpose (Boer et al., 2011), of fitting in, and of being understood and validated by others (e.g., Bernard et al., 2006; Sanderson et al., 2019). For example, individuals who value harmony and social justice (i.e., self-transcendence values) and individuals who value achievement (i.e., self-enhancement value) may be happier if they perceive others to share these values because they feel validated and capable of working to a common goal (i.e., harmony or competition). At the same time however, and as we have seen above, similarity effects differ between value types, suggesting that the motivational processes underlying a particular value type can influence the shape of similarity effects. Future research may benefit from exploring these possibilities further by comparing how the motivational implications of different value types influence value similarity effects, and how they depend on other factors (e.g., availability of resources; norms).

As discussed in the section on romantic relationships, perceived value similarity effects are also likely to involve a range of biases. Misperceptions of other people's values often originate from the inferences that individuals draw from the salient snippets of others' attitudes and behaviours in everyday life (e.g., Hart et al., 2009; Kunda, 1990; cf. Brunswik, 1952). These inferences are biased by selective attention and interpretation serving to confirm our existing views of others, by the media, and by our social circles. For instance, an individual who values protecting the environment may be highly attentive to behaviours from others that damage the environment (e.g., seeing a sports car, litter on the ground), leading the individual to assume that most others do not share their values (Bouman \& Steg, 2019). Hence, such motivational and contextual processes 
may be among the main reasons why we often fail to notice the actual similarities in our values with others (Hanel, Wolfradt, et al., 2018; Hanel \& Wolf, 2019). Nevertheless, as the study reported here shows, perceived value similarities, and the biases they subsume, are worth studying because they capture an important aspect of people's view of their social surroundings in ways that predict their life satisfaction. The extent to which such value similarity effects are underpinned by motivational or cognitive biases in the perceiver is likely to be a fruitful avenue for future research.

This discussion on the extent to which perceived value similarity effects involve biases in the perceiver suggests that perceived and actual value similarities should show marked differences in the patterns and nature of the effects. In fact, recent research from our lab used the PRA and RSA approach to test how actual value similarities between individuals and their region or country predict various well-being outcomes (Hanel et al., 2020). We found that higher similarities in selfdirection, stimulation, and hedonism values (openness values) predict lower well-being, whereas higher similarities in achievement, power (self-enhancement values), and security (conservation value) predict higher well-being across six main indicators. For better comparability, future research may benefit from directly contrasting perceived and actual value similarity effects on wellbeing, using the same reference target and well-being measures.

Finally, there are potential differences in how value similarities relate to well-being at different societal levels, ranging from small communities to towns, cities, regions (e.g., Greater Manchester), countries, continents, and many other cultures and groupings (e.g., LGBTQ groups). Value similarities might also be examined at a group level, comparing the values of one group with another. Research exploring such differences could also consider the role of ingroup identification, given that effects of value similarity may be more pronounced for people who identify more strongly with their country (cf. Abbott et al., 2005). It would also be fruitful to examine the crosscultural aspect of value similarity effects in more detail by including societies beyond a Western context, based on, for instance, considerations that harmony plays a more important role in Eastern cultures. 


\section{Prejudice}

A range of seminal theories has suggested that similarities, and value similarities in particular, are important components of intergroup attitudes (e.g., belief congruence theory, Rokeach et al., 1960; self-categorization theory, Turner et al., 1987; similarity-attraction hypothesis, Byrne, 1961; Byrne \& Wong, 1962). While considerable research attention has been devoted to testing these theories, this research has used absolute difference scores or profile correlations, which are likely to have overstated the role of value similarity as discussed above. Accordingly, previous research has generally provided support for the idea that higher value similarity uniformly predicts lower levels of prejudice. However, this previous work has ignored the possibility that value similarity effects may differ depending on the particular value types under consideration.

In three studies, we aimed to redress these gaps in the literature using the PRA and RSA approach (Wolf et al., 2019). We recruited 350 British online participants to examine how values and value similarities relate to prejudice and symbolic threat against Muslim immigrants, refugees, and economic migrants. We asked participants to indicate their own values and the values they ascribed to Muslim immigrants, refugees, and economic migrants using the Schwartz Value Survey (SVS; Schwartz, 1992) and the Short Schwartz Value Survey (Lindeman \& Verkasalo, 2005). Following Lindeman and Verkasalo (2005), we calculated a score for the self-transcendence versus self-enhancement value dimension and a score for the conservation versus openness value dimension. ${ }^{10}$ Subsequently, we assessed evaluations of the immigrant groups using items assessing liking, trust, and whether participants would support a policy that accepts more immigrants from the group in the country. We also assessed perceptions of symbolic threat from the immigrant groups (Stephan et al., 1999).

10 The Short Schwartz Value Survey (SSVS; Lindeman \& Verkasalo, 2005) used in this research was specifically designed to yield reliable and valid value dimensions, and using the scale in another way (e.g., entering the single items per value type as predictors) would introduce concerns about internal consistency. We calculated value dimension scores for the Schwartz Value Survey (SVS) in a similar way based on evidence by Lindeman and Verkasalo (2005) that the SSVS shows high overlap with the value dimensions as measured by the SVS. 
The three studies provided new evidence that value similarity effects on prejudice emerge on the conservation versus openness dimension, but not on the self-transcendence versus selfenhancement dimension. Follow-up simple slopes analyses also identified that these effects generally emerge at higher levels of immigrant openness values but not at higher levels of immigrant conservation values. That is, when Muslim immigrants, refugees, and economic migrants are perceived to value openness more, British people who hold higher conservation values generally express more negative evaluations and higher perceived threat than British people who hold higher openness values. These (dis)similarity effects when immigrant groups are seen as higher in openness values are consistent with the motivational implications of the respective values. Individuals who attach higher importance to conservation values such as tradition, security, and the status quo may view immigrants with opposing openness values such as freedom and independence as threatening their way of life. In contrast, individuals who attach higher importance to openness values such as curiosity and an exciting life may view immigrants who share their values as particularly likely to satisfy their thirst for something new and unconventional. Similarity effects were consistently absent when these immigrant groups were perceived to be higher in conservation, self-transcendence, and self-enhancement values.

These findings are illustrated in four example plots in Figure 5. The self-transcendence versus self-enhancement value dimension in sections A and B shows consistent linear effects of own and perceived values, such that evaluations of Muslim immigrants and economic migrants are more positive when participants hold higher self-transcendence (versus self-enhancement) values and when they perceive the immigrant groups as being high in self-transcendence (versus selfenhancement) values. ${ }^{11}$ There are no indications of similarity effects. In contrast, the conservation versus openness value dimension in sections $\mathrm{C}$ and $\mathrm{D}$ generally indicates more positive evaluations of the immigrant groups along the similarity line (running from the points $-2 /-2$ to $+2 /+2$ on the

11 These linear effects are small-to-medium and comparable to the similarity effects on the openness versus conservation value dimension. 
predictors), and more negative evaluations close to the dissimilarity points (at $-2 /+2$ and $+2 /-2$ ). The RSA plot in section $\mathrm{C}$ shows similarity effects only at the extreme ends of the predictors (i.e., at $-2 /-2$ and $+2 /+2$ ) due to the absence of significant negative quadratic effects, whereas the plot in section $\mathrm{D}$ is somewhat askew due to the influence of a negative linear effect of own conservation values.

A

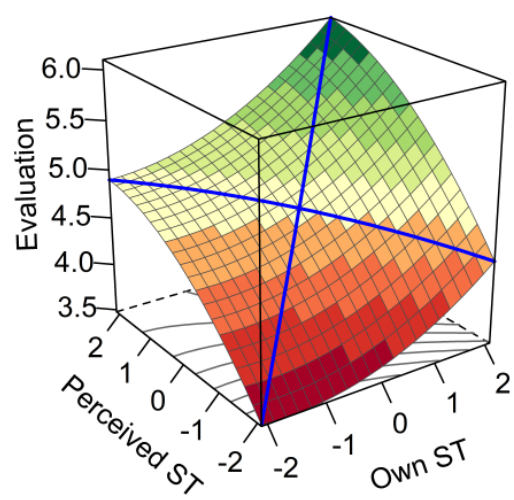

C

\section{Muslim immigrants}

Muslim immigrants

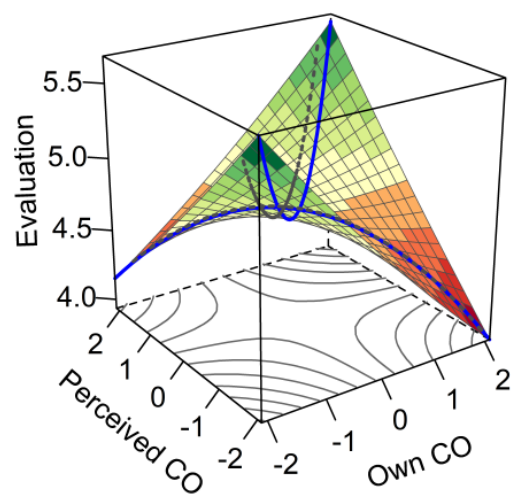

Economic migrants

B

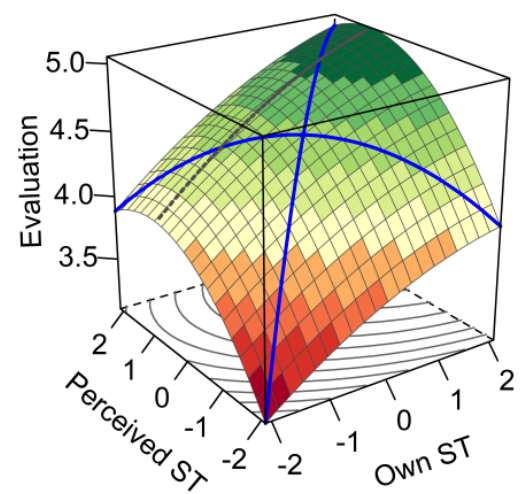

Refugees

D

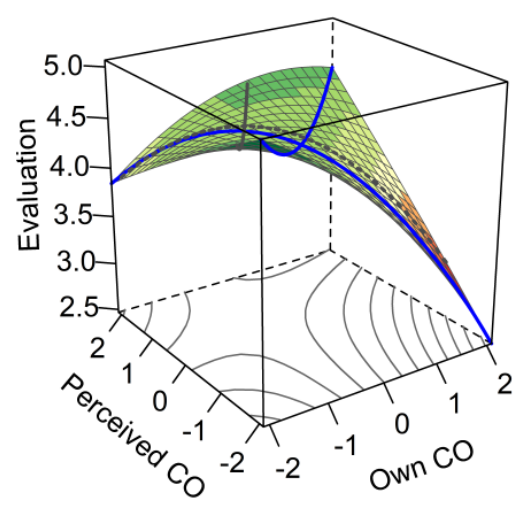

Figure 5. Response surface analyses plots illustrating effects of own and perceived selftranscendence versus self-enhancement values ("ST"; sections A and B) and effects of own and perceived conservation vs openness values (“CO”; sections C and D) on evaluations of immigrant groups. Higher positive scores on the predictors indicate higher self-transcendence or conservation values whereas higher negative scores indicate higher self-enhancement or openness values. 
While the potential for greater intergroup positivity from value matches was revealed along the openness to conservation dimension, its absence on the self-transcendence-to-self-enhancement dimension is intriguing. We speculate that this absence may stem from effects of own and perceived values on this dimension that are too strong to be superseded by a value similarity effect. In other words, value similarity may not provide additional stimulants for higher favourability among individuals who are already strongly predisposed through their values to like immigrants and who already see immigrants' values favourably. Future research may benefit from examining this speculation more closely. For instance, it could be tested whether dissimilarity effects on this value dimension are only absent when assessed indirectly (e.g., when participants provide assessments of own and perceived values and similarities as established through a PRA and RSA approach), or whether similarity also does not provide additional favourability when assessed directly (e.g., "how similar is the outgroup's benevolence value to yours?").

Overall, then, this research shows that effects of value similarity on intergroup attitudes depend greatly on which values are examined, partially contradicting previous theories and research which suggest that value similarity uniformly predicts lower levels of prejudice. It is important to note, however, that the findings are consistent in a broad sense with seminal theories in the prejudice literature that together suggest a more nuanced pattern for similarity effects. On the one hand, self-categorization theory (Turner et al., 1987), belief congruence theory (Rokeach et al., 1960), and similarity-attraction theory (Byrne, 1961; Byrne \& Wong, 1962) relate higher value similarity to lower prejudice. On the other hand, social identity theory (Tajfel \& Turner, 1979) and optimal distinctiveness theory (Brewer, 1991) indicate that too high levels of similarity with others and other groups can be seen as threatening one's or the ingroup's sense of uniqueness. Jetten et al. (2004) have highlighted these conflicting perspectives and shown that both predictions are valid, with one or the other effect emerging depending on the particular circumstances (i.e., identification, outcome measure). 
These conflicting predictions may also apply to the present research. While higher value similarity may indeed generally predict lower prejudice, this pattern could have been masked because individuals felt that their group identity was threatened at higher levels of value similarity with an outgroup. This may explain the absence of similarity effects when immigrants were perceived to be higher in conservation values, self-transcendence values, and self-enhancement values. In contrast, individuals higher in openness values still show a value similarity effect, perhaps because high value similarity with immigrants is perceived as less threatening for their group identity. This explanation is based on findings that individuals who value openness are less identified with their ingroup (Roccas et al., 2010), and among those less identified, similarity is experienced as less threatening such that higher similarity results in lower prejudice (Jetten et al., 2004). Hence, the present findings supports previous assertions that higher value similarity predicts less prejudice but only when high similarity is not perceived as threatening, as appears to be the case among those higher in openness values. It would be fruitful to examine these explanations for differential value dissimilarity effects more closely in future research, for instance by directly testing the role of ingroup identification and similarity threat. As in research on well-being, further complexities may arise from (a) the particular reference group, (b) whether self-outgroup or ingroup-outgroup value similarities are considered, and (c) the extent to which resources are scarce and could elicit goal conflict. These complexities provide interesting avenues for future research to explore.

In short, by using an improved analytical method, PRA and RSA, these three studies revealed a picture for dissimilarity effects on prejudice that provides a new level of nuance, but one that is more in line with seminal theories (e.g., Brewer, 1991; Tajfel \& Turner, 1979) and with previous suggestions that dissimilarity does not always predict higher prejudice (e.g., Jetten et al., 2004). 


\section{Linking Perceived Value Similarity Effects Across Outcomes}

In broader terms, it is important to note that the perceived similarity effects of openness values on prejudice are consistent with the Manchester findings for perceived value similarity in openness values on well-being. Across all four studies, participants reported higher well-being and lower prejudice when their own openness values aligned with the perceived values of fellow citizens and immigrants. Although the Manchester study additionally found similarity effects for self-transcendence and self-enhancement on well-being, these effects may have been absent in the studies on prejudice due to strong linear effects as discussed above; this broad consistency may suggest that value similarity effects are comparable across outcomes to some extent.

This comparability between value similarity effects on prejudice and well-being is also apparent in considerations of their underlying mechanisms which may apply across outcomes. Value similarity effects may occur because similar reference targets (e.g., fellow citizens, immigrants) allow people to express their values and feel understood and validated (Sanderson et al., 2019), thereby potentially increasing personal well-being and reducing prejudice (Solomon \& Knafo-Noam, 2007). Moreover, people who embrace the prevailing norms and values are commonly rewarded in society, allowing them to attain their values and goals, whereas people who reject the norms and values are often sanctioned, blocking their values and goals, which is likely to have repercussions for people's well-being and potentially their attitudes toward others. These mechanisms may also apply to perceived value similarity effects in romantic relationships and organisations, where a similar partner, supervisor, or colleague promotes feelings of being understood and facilitates goal attainment, which should result in higher satisfaction. Further research is needed to examine these underlying mechanisms in the context of value similarity effects on well-being and prejudice.

Additional outcomes beyond well-being and prejudice may warrant further research attention. As mentioned at the outset of this review, shared values are key binding factors that may facilitate social belongingness, shared norms, and positive intergroup and interpersonal relations, 
and we therefore expect value similarities to affect a range of important outcomes within and across societies. One such outcome may be compliance to Covid-19 guidelines. That is, as argued in a recent article from our lab (Wolf et al., 2020), perceiving fellow citizens to share one's values may motivate individuals to engage in behaviour compliant with Covid-19 guidelines (e.g., wearing masks in public, keeping $2 \mathrm{~m}$ distance), because the sense of common purpose arising from perceptions of shared values may facilitate a concerted, collective response. In contrast, perceiving others to have different values may hinder the belief that a collective response from the public is feasible and effective, potentially reducing people's willingness to self-sacrifice for the greater societal good. Based on this reasoning, we provide recommendations for developing global interventions to tackle the Covid-19 pandemic. Moreover, similar considerations likely apply to other pro-social (e.g., communities supporting each other) and pro-environmental behaviours (e.g., reducing travel, littering) in societies, where perceiving shared values may often predict more positive outcomes. It is worth noting, however, that for some outcomes, perceived dissimilarities rather than similarities may be more beneficial. For instance, when the goal is to think outside the box in a group meeting or to counteract phenomena such as social loafing, groupthink, or deindividuation in groups, it could be more advantageous to focus on what sets people apart. The question of how value similarity effects extend to other outcomes beyond well-being and prejudice is likely a fruitful avenue for future research to explore.

Another important issue relates to how immigrants' perceptions of value similarities relate to how they are acculturated in their host country. Acculturation refers to a bilateral process of change as a result of the encounter of two or more cultures (Berry, 1997). Applied to the present context, when immigrants arrive in another country, they might initially feel a "cultural shock" which includes feelings of value dissimilarity (Stromberg \& Boehnke, 2001). Value similarities are important because they may predict acculturation strategies (e.g., Schiefer et al., 2012), which in turn are relevant for immigrants' mental health (Yoon et al., 2013). The acculturation strategy "integration", which refers to identifying with the host culture while still identifying with the home 
culture (i.e., acknowledging similarities between home and host culture; Berry, 1997), is particularly strongly linked with higher immigrant well-being (e.g., Berry et al., 2006; Yoon et al., 2013). The underlying mechanisms for effects of value similarities on acculturation may be similar to those for well-being (Stromberg \& Boehnke, 2011), including, for example, the absence of social sanctions and internal conflicts (Sagiv \& Schwartz, 2000). However, to the best of our knowledge, no published study has investigated the effects of value similarities on acculturation or well-being among immigrants using polynomial regression analysis.

\section{Causal Effects of Value Similarity}

The research we have reviewed in the previous sections drew on cross-sectional data. Such research is essential to understand how naturally occurring differences in value similarity are linked with outcomes such as well-being and prejudice, and this ecologically valid approach has generally shown that higher similarity in certain values relates to more positive outcomes. However, these findings also raise the question about the directionality of effects and their underlying causal mechanisms. Does higher value similarity cause more positive outcomes or does, for instance, higher well-being lead people to assume that they are more similar to others around them? Does higher value similarity increase people's sense of being understood and validated as speculated above? To examine such questions, studies employing experimental manipulations of value similarity are essential.

Vione (2016) found that experimentally highlighting similarities in values on an individual level can influence how other individuals are perceived. Specifically, she first measured student participants' values on the SVS and used their responses to create hypothetical profiles that either matched their values at $100 \%$ (i.e., identical), $82 \%$, or $64 \%$. In a later session, these profiles were presented to participants as fellow students who were of similar age and gender but from a different university class. Participants then completed measures assessing their general attitudes towards the target, cognitive evaluations (e.g., whether the target is well-respected), perceptions of the target's warmth and competence, and interpersonal attraction. Comparing the $100 \%$ and $64 \%$ matching 
conditions, Vione found that describing another person's values as identical to participants' own values increased perceived warmth and attraction compared to describing the person's values as moderately similar. No effects on the other dependent variables were found. This research hence shows that higher value similarity can indeed cause more positive perceptions and motivations towards others, a finding broadly consistent with seminal evidence showing that higher value similarity causes a reduction in prejudice (Rokeach \& Mezei, 1966; Stein et al., 1965), higher organisational commitment (Bai et al., 2017) and organisational identification (Edwards \& Cable, 2009).

Nonetheless, a limitation of Vione's (2016) approach is the use of fictitious hypothetical value similarity information instead of real data on others' values to manipulate the information. We have recently tested whether highlighting actual value similarities based on real data can affect intergroup favourability, a possibility that emerges from evidence across a range of countries that people often misperceive others' values (Bernard et al., 2006; Fischer, 2006). For example, Hanel, Wolfradt, et al. (2018) found that three out of four people valued self-transcendence more than selfenhancement, but only one out of four perceived their fellow citizens to have a similar value priority. When people misperceive the values of those living in the same city or country, it is likely that they also misperceive the values of people they know less, such as immigrants. However, it is unclear what happens if these misperceptions are rectified.

We began to address this question in a series of experimental studies. Our approach was based on the observation that the common statistical methods for comparing groups (e.g., British and Polish people) are biased towards differences, while similarities are widely ignored (Hanel, Maio, \& Manstead, 2019). Instead of solely focusing on mean differences, as is commonly done in statistics (e.g., t-tests), we considered all individual responses to estimate the amount of similarities between groups. For example, we found that the amount of similarity or overlap in values between British and Polish people is around $88 \%$ - even though many of the pairwise comparisons reached statistical significance and are of small-to-medium size. Figure 6 displays ratings of the importance 
of "tradition" values of representative samples from Poland $(n=1615)$ and the UK $(n=2264$; data came from the European Social Survey, $7^{\text {th }}$ round): The left graph is commonly used in psychology to display research findings (here: Cohen's $d=0.40$ ). The right graph, however, shows that the similarities between both groups outweigh the differences ( $84 \%$ similarity). To create the graphs, we used the actual means, standard deviations, and sample sizes. Overall, we found that similarities among various groups of people (e.g., across different countries, educational levels, gender) range between $80 \%$ and $95 \%$ on average across psychological variables such as values and attitudes.
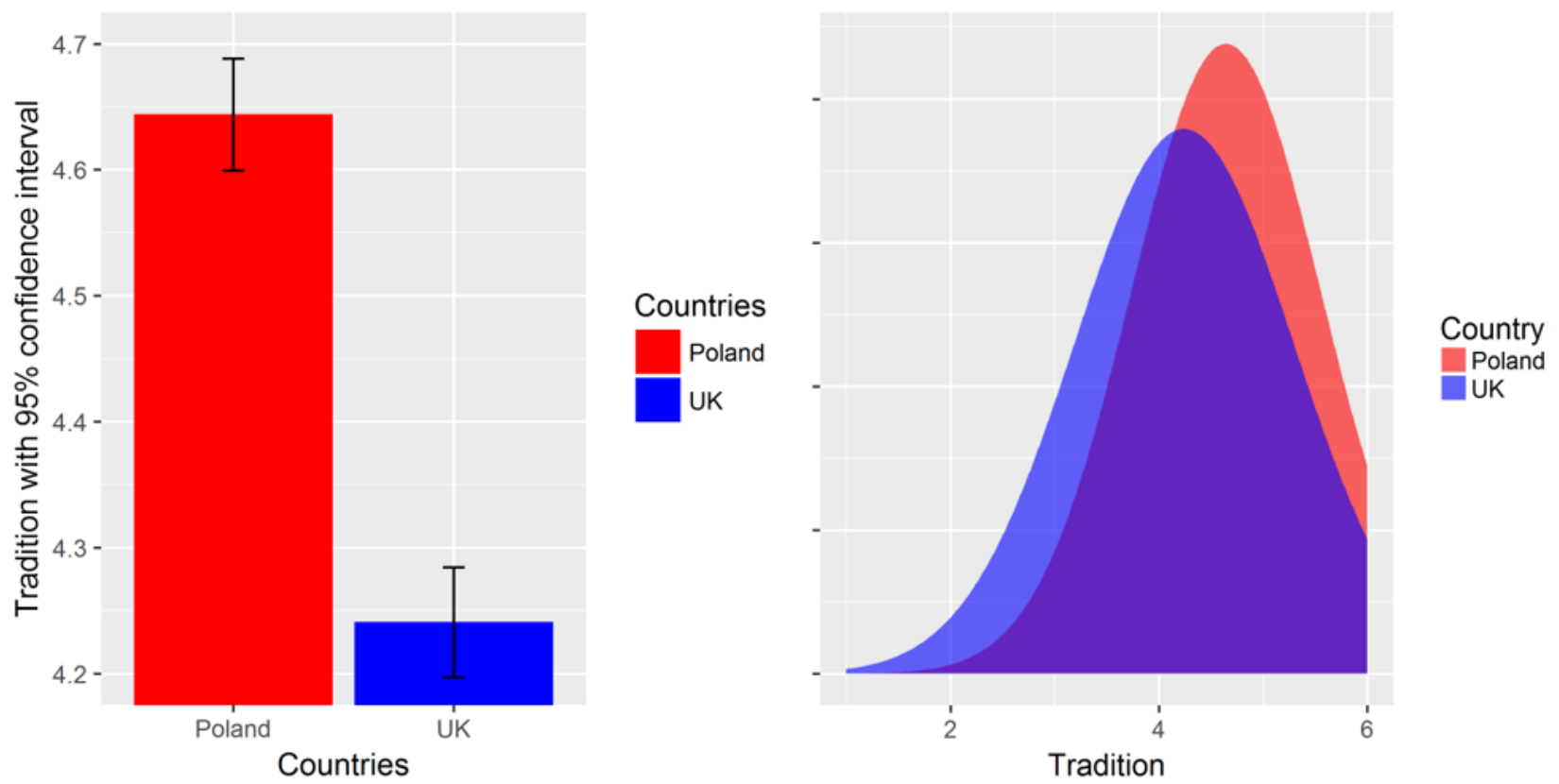

Figure 6. Standard presentation of results (left-panel) that emphasises differences vs a graph that highlights similarities (right-panel).

Across three further studies, we presented participants with information about values in a manner that highlighted actual similarities using superimposed normal distributions (Figure 6, right graph) or radarcharts, or we presented the same information using more typical barplots with restricted y-axes, which emphasise differences (Figure 6, left panel). We found that normal distributions and radarplots cause more positive intergroup attitudes among British and Polish people and among Leave and Remain voters in the Brexit referendum (Hanel \& Wolf, 2019) than barplot presentations. A particular strength of this graphical approach in highlighting similarities is 
that it facilitates the presentation of information about large groups of participants, thus preventing group subtyping, which is a psychological response often used to protect stereotypes (Richards \& Hewstone, 2001). Take mathematical abilities as an example. Meta-analytical research established that women and men have similar mathematical abilities on average (Hyde et al., 1990; Hyde et al., 2008). Highlighting a few mathematically talented women might not increase the perception that women and men are equal, but rather that there are a few exceptional female mathematical geniuses. Instead, when we superimpose two normal distributions reflecting mathematical abilities of women and men (similar to Fig. 6, right panel; see also Hyde et al., 1990), it becomes apparent that mathematical abilities are $>98 \%$ similar between women and men. Further, unlike previous research that found positive effects of highlighting similarities on intergroup harmony, we presented the same data in both experimental conditions, but only changed the mode of presentation.

The findings additionally showed that people's ability to estimate the actual effect size between two groups is improved when presented with normal distributions compared to barplots with restricted y-axes (Figure 6), even though participants in the latter conditions were informed about the full range of the response scale (e.g., 1 to 6). Further, people rate the similarity effect size we used as more informative than Cohen's $d$ (Hanel, Maio, \& Manstead, 2019; Hanel \& Mehler, 2019) and of greater potential relevance to practitioners (Satchell, 2019). Hence, this method of comparing groups using normal distributions is a novel and promising tool to promote intergroup positivity while improving accuracy and perceived utility among the public and practitioners.

There are at least three untested mechanisms that can explain why highlighting value similarities in normal distributions improves intergroup attitudes. First, highlighting similarities alters previous, negative misperceptions of the values of the outgroup (Hanel, Maio, \& Manstead, 2019). Rectifying these misperceptions may hence improve the negative evaluations of the outgroup. Second, by displaying superimposed normal distributions, not only are similarities highlighted but also the heterogeneity of both the in- and outgroup (the variability of values is usually similar among countries; Hanel, Zarzeczna, \& Haddock, 2019). This presentation of 
heterogeneity is important because outgroups that are presented as heterogeneous are treated more fairly than outgroups that are perceived as homogeneous (Brauer \& Er-rafiy, 2011; Vandeselaere, 1991). Finally, highlighting similarities might reduce the boundaries between the in- and outgroup. This prediction is based on social identity theory (Tajfel \& Turner, 1979) and the common ingroup identity model (Gaertner \& Dovidio, 2000). The latter model postulates that, if the boundaries between the in- and outgroup become less distinct or vanish, prejudice decreases. Thus, plotting superimposed distributions of the data may be beneficial for multiple reasons.

It would be timely to begin using this method to examine other potential outcomes of value similarity. In particular, it is conceivable that the quality of debate between polarised groups (e.g., political, religious, ethnic groups) is less ad hominem and more epistemically genuine when the opposing groups are shown evidence of greater value similarity than believed. This evidence might motivate people to engage in more perspective taking and encourage listening to the opposing group (e.g., Itzchakov et al., 2017). There are also potential implications for improving intercultural communication, where people from other countries are often displayed as different and homogeneous. While certain behavioural differences are important (e.g., shaking hands vs bowing), highlighting similarities and outgroup heterogeneity could for instance be beneficial for business relations by acknowledging that, although some behaviours differ, the underlying values are generally similar and as heterogeneous as the values of the in-group.

In fact, the context of intercultural communication helps to point out an important theoretical complication in examining similarities and differences between values. Specifically, as mentioned before, mental representations of values also subsume diverse concrete instantiations (Maio, 2010). People learn conceptual categories through experience, such that individuals' personal and social experience influences which actions and situations are seen as relevant to each value (Hanel et al., 2017). These experiences are nested within individuals' social-cultural milieu, which further curtails the actions and situations that are used to exemplify the values. As a result, instantiations of values can vary in many ways. For example, the value of equality can subsume 
different targets (e.g., gender, sexual orientation, ethnicity, body size), actions (e.g., employment decisions, sex roles), and contexts (e.g., public, private). To illustrate, the broad value of equality is rated similarly in importance between European Union countries and Turkey, but perceptions of its specific relevance to gender are strikingly different (Hanel et al., 2017). Such differences in instantiating values are important because egalitarian behaviour is affected not only by the salience of the value to individuals, but also by the salience of instantiations of the value that are typical (vs atypical) in their cultural environment (Maio, Hahn, et al., 2009).

These considerations raise an important question: What would happen if people learned about other groups' values and value instantiations? Information about value similarities should increase liking, but the effect of value similarity may also depend on individuals' beliefs about the specific value instantiations. Learning about value similarity might have more impact if people also learn that the outgroup's instantiations are more similar to their own than believed. For example, although the value of social justice may be instantiated differently between European Union countries and Turkey, a manipulation could highlight that people across these countries reject theft as unfair and as violating social justice (Hanel, Maio, \& Manstead, 2019). It is important to evaluate these possibilities because the delivery of qualitative descriptions of the values may be a key component of any real-world intervention focused on values. By enriching the abstract definition of values with more concrete descriptions of how different groups understand and strive to apply the values, higher levels of engagement and understanding may be sustained, with a potential impact on attitudes and intercultural communication. From this perspective, the information delivery may be viewed as a kind of informational intergroup contact. Contact between members of different groups promotes more positive intergroup attitudes (Pettigrew \& Tropp, 2006), even when the contact is merely imagined (Crisp \& Turner, 2009). Learning about other groups' real values and how they instantiate these may come close to the experience of actual contact, through the knowledge that group members are directly contributing the information. 


\section{Conclusion}

In this review, we have discussed the importance of value similarities for well-being and prejudice. We reviewed several cross-sectional studies which used a statistically more precise approach, polynomial regressions and response surface analyses, to examine value similarity effects. These studies showed that well-being and prejudice against outgroups often depend on whether people's own and others' values (are perceived to) align, and importantly, that such effects differ depending on the particular values under consideration. For instance, individuals report higher well-being and lower prejudice when their own openness values align with the perceived values of fellow citizens and immigrants. The findings reveal a consistent role for value similarity effects in openness values, perhaps because individuals who give importance to openness values such as curiosity and an exciting life may find like-minded others particularly enjoyable.

Further, we have reviewed experimental evidence that value similarities can be manipulated using an easy-to-implement method which rectifies people's misperceptions of others' values and results in more positive attitudes towards the outgroup. When people are made aware that outgroup members are actually similar to them, the boundaries to the outgroup members may become more fuzzy. This research used a novel technique to present information about how groups compare, and this technique was perceived as more informative and useful by the public. Hence, more frequent and more widespread use of this technique in the public domain may improve intergroup harmony among groups and improve accuracy of the public understanding of group similarities and difference. Overall, we hope that the presented research, their novel and statistically more precise methods, and the discussions of underlying mechanisms spark further research into when and how value similarity effects emerge, which types of outcomes they predict, and the greater use of simple interventions that can improve public well-being and intergroup attitude. 


\section{References}

Abbott, G. N., White, F. A., \& Charles, M. A. (2005). Linking values and organizational commitment: A correlational and experimental investigation in two organizations. Journal of Occupational and Organizational Psychology, 78(4), 531-551. https://doi.org/10.1348/096317905X26174

Agnew, C., Lange, P. V., Rusbult, C., \& Langston, C. (1998). Cognitive Interdependence: Commitment and the Mental Representation of Close Relationships. Journal of Personality and Social Psychology, 74(4), 939-954.

Allport, G. W. (1954). The nature of prejudice. Cambridge, MA: Addison-Wesley.

Aryamanesh, S., Fallahchai, R., Zarei, E., \& Haghighi, H. (2012). Comparison of differentiation among satisfying couples and conflicting couples in Bandar Abbas city. Journal of Life Science and Biomedicine, 2(6), 308-314.

Assouline, M., \& Meir, E. I. (1987). Meta-analysis of the relationship between congruence and well-being measures. Journal of Vocational Behavior, 31(3), 319-332. https://doi.org/10.1016/0001-8791(87)90046-7

Bai, Y., Dong, Z., Liu, H., \& Liu, S. (2017). We may be different, but I can help you: The effects of leaders' political skills on leader-follower power distance value incongruence and withdrawal behavior. Journal of Leadership \& Organizational Studies, 24(2), 216-229. https://doi.org/10.1177/1548051816665582

Barranti, M., Carlson, E. N., \& Côté, S. (2017). How to test questions about similarity in personality and social psychology research Description and empirical demonstration of response surface analysis. Social Psychological and Personality Science, 8(4), 465-475. https://doi.org/10.1177/1948550617698204 
Benish-Weisman, M. , Daniel, E. , \& McDonald, K. (2019). Values and adolescents' self-esteem: The role of value content and congruence with classmates. European Journal of Social Psychology, 50, 207-223. https://doi.org/10.1002/ejsp.2602

Bernard, M. M., Gebauer, J. E., \& Maio, G. R. (2006). Cultural estrangement: The role of personal and societal value discrepancies. Personality and Social Psychology Bulletin, 32(1), 78-92. https://doi.org/10.1177/0146167205279908

Berry, J. W. (1997). Immigration, acculturation, and adaptation. Applied Psychology, 46(1), 5-34. https://doi.org/10.1111/j.1464-0597.1997.tb01087.x

Berry, J. W., Phinney, J. S., Sam, D. L., \& Vedder, P. (2006). Immigrant Youth: Acculturation, Identity, and Adaptation. Applied Psychology, 55(3), 303-332. https://doi.org/10.1111/j.1464-0597.2006.00256.x

Bilsky, W., Janik, M., \& Schwartz, S. H. (2011). The structural organization of human valuesEvidence from three rounds of the European Social Survey (ESS). Journal of CrossCultural Psychology, 42(5), 759-776. https://doi.org/10.1177/0022022110362757

Bleidorn, W., Schönbrodt, F., Gebauer, J. E., Rentfrow, P. J., Potter, J., \& Gosling, S. D. (2016). To live among like-minded others: Exploring the links between person-city personality fit and self-esteem. Psychological Science, 27(3), 419-427. https://doi.org/10.1177/0956797615627133

Boer, D., Fischer, R., Strack, M., Bond, M. H., Lo, E., \& Lam, J. (2011). How shared preferences in music create bonds between people: Values as the missing link. Personality and Social Psychology Bulletin, 37(9), 1159-1171. https://doi.org/10.1177/0146167211407521

Bouman, T., \& Steg, L. (2019). Motivating Society-wide Pro-environmental Change. One Earth, 1(1), 27-30.

Brauer, M., \& Er-rafiy, A. (2011). Increasing perceived variability reduces prejudice and discrimination. Journal of Experimental Social Psychology, 47(5), 871-881. https://doi.org/10.1016/j.jesp.2011.03.003 
Brewer, M. B. (1991). The social self: On being the same and different at the same time.

Personality and Social Psychology Bulletin, 17(5), 475-482.

https://doi.org/10.1177/0146167291175001

Brunswik, E. (1952). The conceptual framework of psychology. University of Chicago Press.

Byrne, D. (1961). Interpersonal attraction and attitude similarity. The Journal of Abnormal and Social Psychology, 62(3), 713-715. https://doi.org/10.1037/h0044721

Byrne, D., \& Wong, T. J. (1962). Racial prejudice, interpersonal attraction, and assumed dissimilarity of attitudes. The Journal of Abnormal and Social Psychology, 65(4), 246-253. https://doi.org/10.1037/h0047299

Chatman, J. A. (1989). Matching people and organizations: Selection and socialization in public accounting firms. Academy of Management Proceedings, 1989(1), 199-203. https://doi.org/10.5465/ambpp.1989.4980837

Coelho, G. L. de H., Hanel, P. H. P., Johansen, M., \& Maio, G. R. (2019). Mapping the structure of human values through conceptual representations. European Journal of Personality, 33(1), 34-51. https://doi.org/10.1002/per.2170

Condon, J. W., \& Crano, W. D. (1988). Inferred evaluation and the relation between attitude similarity and interpersonal attraction. Journal of Personality and Social Psychology, 54(5), 789-797. https://doi.org/10.1037/0022-3514.54.5.789

Crisp, R. J., \& Turner, R. N. (2009). Can imagined interactions produce positive perceptions? Reducing prejudice through simulated social contact. American Psychologist, 64(4), 231240. https://doi.org/10.1037/a0014718

Davidov, E., Meuleman, B., Billiet, J., \& Schmidt, P. (2008). Values and support for immigration: A cross-country comparison. European Sociological Review, 24(5), 583-599. https://doi.org/10.1093/esr/jcn020

Doolittle, A., \& Faul, A. C. (2013). Civic engagement scale: A validation study. SAGE Open, 3(3), 2158244013495542. https://doi.org/10.1177/2158244013495542 
Du, H., Chen, A., Chi, P., \& King, R. B. (2019). Person-culture fit boosts national pride: A crosscultural study among 78 countries. Journal of Research in Personality. https://doi.org/10.1016/j.jrp.2019.05.008

Dyrenforth, P. S., Kashy, D. A., Donnellan, M. B., \& Lucas, R. E. (2010). Predicting relationship and life satisfaction from personality in nationally representative samples from three countries: The relative importance of actor, partner, and similarity effects. Journal of Personality and Social Psychology, 99(4), 690-702. https://doi.org/10.1037/a0020385

Edwards, J. R. (1993). Problems with the use of profile similarity indices in the study of congruence in organizational research. Personnel Psychology, 46(3), 641-665. https://doi.org/10.1111/j.1744-6570.1993.tb00889.x

Edwards, J. R. (1994). The study of congruence in organizational behavior research: Critique and a proposed alternative. Organizational Behavior and Human Decision Processes, 58(1), 51100. https://doi.org/10.1006/obhd.1994.1029

Edwards, J. R. (2002). Alternatives to difference scores: Polynomial regression analysis and response surface methodology. In F. Drasgow \& N. W. Schmitt (Eds.), Advances in measurement and data analysis (pp. 350-400). San Francisco, CA: Jossey-Bass.

Edwards, J. R. (2008). Person-environment fit in organizations: An assessment of theoretical progress. The Academy of Management Annals, 2(1), 167-230. https://doi. org/10.1080/19416520802211503.

Edwards, J. R., \& Cable, D. M. (2009). The value of value congruence. Journal of Applied Psychology, 94(3), 654-677. https://doi.org/10.1037/a0014891

Edwards, J. R., \& Cooper, C. L. (1990). The person-environment fit approach to stress: Recurring problems and some suggested solutions. Journal of Organizational Behavior, 11(4), 293307. https://doi.org/10.1002/job.4030110405 
Fischer, R. (2006). Congruence and functions of personal and cultural values: Do my values reflect my culture's values? Personality and Social Psychology Bulletin, 32(11), 1419-1431. https://doi.org/10.1177/0146167206291425

Fischer, R. (2017). From values to behavior and from behavior to values. In S. Roccas \& S. Lilach (Eds.), Values and behaviour: Taking a cross-cultural perspective (pp. 219-235). Heidelberg: Springer.

Fitzsimons, G. M., \& Fishbach, A. (2010). Shifting closeness: Interpersonal effects of personal goal progress. Journal of Personality and Social Psychology, 98(4), 535-549. https://doi.org/10.1037/a0018581

Gaertner, S. L., \& Dovidio, J. F. (2000). Reducing intergroup bias: The common ingroup identity model. Philadelphia, PA: Psychology Press.

Gagné, F. M., \& Lydon, J. E. (2004). Bias and accuracy in close relationships: An integrative review. Personality and Social Psychology Review, 8(4), 322-338. https://doi.org/10.1207/s15327957pspr0804_1

Gere, J., Schimmack, U., Pinkus, R. T., \& Lockwood, P. (2011). The effects of romantic partners' goal congruence on affective well-being. Journal of Research in Personality, 45(6), 549559. https://doi.org/10.1016/j.jrp.2011.06.010

Griffin, D., Murray, S., \& Gonzalez, R. (1999). Difference score correlations in relationship research: A conceptual primer. Personal Relationships, 6(4), 505-518. https://doi.org/10.1111/j.1475-6811.1999.tb00206.x

Hanel, P. H. P., Litzellachner, L. F., \& Maio, G. R. (2018). An empirical comparison of human value models. Frontiers in Psychology, 9. https://doi.org/10.3389/fpsyg.2018.01643

Hanel, P. H. P., Maio, G. R., \& Manstead, A. S. R. (2019). A new way to look at the data: Similarities between groups of people are large and important. Journal of Personality and Social Psychology, 116(4), 541-562. https://doi.org/10.1037/pspi0000154 
Hanel, P. H. P., \& Mehler, D. M. (2019). Beyond reporting statistical significance: Identifying informative effect sizes to improve scientific communication: Public Understanding of Science, 28(4), 468-485. https://doi.org/10.1177/0963662519834193

Hanel, P. H. P., Vione, K. C., Hahn, U., \& Maio, G. R. (2017). Value instantiations: The missing link between values and behavior? In S. Roccas \& S. Lilach (Eds.), Values and behaviour: Taking a cross-cultural perspective (pp. 175-190). Heidelberg: Springer.

Hanel, P. H. P., \& Wolf, L. J. (2019). Leavers and Remainers after Brexit: More united than divided after all? British Journal of Social Psychology. Online first publication. https://doi.org/10.1111/bjso.12359.

Hanel, P. H. P., Wolfradt, U., Coelho, G. L. de H., Wolf, L. J., Vilar, R., Monteiro, R. P., ... Maio, G. R. (2018). The perception of family, city, and country values is often biased. Journal of Cross-Cultural Psychology, 49(5), 831-850. https://doi.org/10.1177/0022022118767574

Hanel, P. H. P., Wolfradt, U., Wolf, L. J., Coelho, G. L. de H., \& Maio, G. R. (2020). Well-being as a function of person-country fit in human values. Nature Communications. Accepted in principle.

Hanel, P. H. P., Zarzeczna, N., \& Haddock, G. (2019). Sharing the same political ideology yet endorsing different values: Left-and right-wing political supporters are more heterogeneous than moderates. Social Psychological and Personality Science, 10(7), 874-882. https://doi.org/10.1177/1948550618803348

Hart, W., Albarracín, D., Eagly, A. H., Brechan, I., Lindberg, M. J., \& Merrill, L. (2009).

Feeling validated versus being correct: A meta-analysis of selective exposure to information. Psychological Bulletin, 135, 555-588. https://doi.org/10.1037/a0015701

Hermans, H. J. M., \& Oles, P. K. (1996). Value crisis: Affective organization of personal meanings. Journal of Research in Personality, 30(4), 457-482. https://doi.org/10.1006/jrpe.1996.0034 
Higgins, E. T., Shah, J., \& Friedman, R. (1997). Emotional responses to goal attainment: Strength of regulatory focus as moderator. Journal of Personality and Social Psychology, 72, 515525. https://doi.org/10.1037/0022-3514.72.3.515

Hitlin, S., \& Piliavin, J. A. (2004). Values: Reviving a dormant concept. Annual Review of Sociology, 30(1), 359-393. https://doi.org/10.1146/annurev.soc.30.012703.110640

Holland, J. L. (1997). Making vocational choices: A theory of vocational personalities and work environments, 3rd ed (3rd ed.). Odessa, FL: Psychological Assessment Resources.

Humberg, S., Nestler, S., \& Back, M. D. (2018). Response surface analysis in personality and social psychology: Checklist and clarifications for the case of congruence hypotheses. Social Psychological and Personality Science, 1948550618757600. https://doi.org/10.1177/1948550618757600

Hyde, J. S., Fennema, E., \& Lamon, S. J. (1990). Gender differences in mathematics performance: A meta-analysis. Psychological Bulletin, 107(2), 139-155. https://doi.org/10.1037/00332909.107.2.139

Hyde, J. S., Lindberg, S. M., Linn, M. C., Ellis, A. B., \& Williams, C. C. (2008). Gender similarities characterize math performance. Science, 321(5888), 494-495. https://doi.org/10.1126/science.1160364

Itzchakov, G., Kluger, A. N., \& Castro, D. R. (2017). I am aware of my inconsistencies but can tolerate them: The effect of high quality listening on speakers' attitude ambivalence. Personality and Social Psychology Bulletin, 43(1), 105-120. https://doi.org/10.1177/0146167216675339

James, L. R. (1982). Aggregation bias in estimates of perceptual agreement. Journal of Applied Psychology, 67(2), 219-229. https://doi.org/10.1037/0021-9010.67.2.219

Jetten, J., Spears, R., \& Postmes, T. (2004). Intergroup distinctiveness and differentiation: A metaanalytic integration. Journal of Personality and Social Psychology, 86(6), 862-879. https://doi.org/10.1037/0022-3514.86.6.862 
Joyce, W. F., \& Slocum, J. W. (1984). Collective Climate: Agreement as a Basis for Defining Aggregate Climates in Organizations. Academy of Management Journal, 27(4), 721-742. https://doi.org/10.5465/255875

Kelley, Harold H., \& Thibaut, J. W. (1978). Interpersonal relations: A theory of interdependence. New York: Wiley-Interscience.

Khaptsova, A., \& Schwartz, S. H. (2016). Life satisfaction and value congruence: Moderators and extension to constructed socio-demographic groups in a russian national sample. Social Psychology, 47(3), 163-173. https://doi.org/10.1027/1864-9335/a000268

Kinder, D. R., \& Sears, D. O. (1981). Prejudice and politics: Symbolic racism versus racial threats to the good life. Journal of Personality and Social Psychology, 40(3), 414-431. https://doi.org/10.1037/0022-3514.40.3.414

Knight, E. L., \& Mehta, P. H. (2017). Hierarchy stability moderates the effect of status on stress and performance in humans. Proceedings of the National Academy of Sciences, 114(1), 78-83. https://doi.org/10.1073/pnas.1609811114

Kristof-Brown, A. L., Zimmerman, R. D., \& Johnson, E. C. (2005). Consequences of Individuals' Fit at Work: A Meta-Analysis of Person-Job, Person-Organization, Person-Group, and Person-Supervisor Fit. Personnel Psychology, 58(2), 281-342. https://doi.org/10.1111/j.1744-6570.2005.00672.x

Kunda, Z. (1990). The case for motivated reasoning. Psychological Bulletin, 108, 480-498. http://dx.doi.org/10.1037/0033-2909.108.3.480

Lee, K., Ashton, M. C., Pozzebon, J. A., Visser, B. A., Bourdage, J. S., \& Ogunfowora, B. (2009). Similarity and assumed similarity in personality reports of well-acquainted persons. Journal of Personality and Social Psychology, 96, 460-472. https://doi.org/10.1037/a0014059

Leikas, S., Ilmarinen, V.-J., Verkasalo, M., Vartiainen, H.-L., \& Lönnqvist, J.-E. (2018). Relationship satisfaction and similarity of personality traits, personal values, and attitudes. 
Personality and Individual Differences, 123, 191-198.

https://doi.org/10.1016/j.paid.2017.11.024

Lindeman, M., \& Verkasalo, M. (2005). Measuring values with the short Schwartz's Value Survey. Journal of Personality Assessment, 85(2), 170-178.

https://doi.org/10.1207/s15327752jpa8502_09

Maio, G. R. (2010). Mental representations of social values. In M. P. Zanna (Ed.), Advances in Experimental Social Psychology, Vol. 42 (Vol. 42, pp. 1-43). San Diego, CA: Academic Press.

Maio, G. R. (2016). The psychology of human values. London: Psychology Press.

Maio, G. R., Hahn, U., Frost, J.-M., \& Cheung, W.-Y. (2009). Applying the value of equality unequally: Effects of value instantiations that vary in typicality. Journal of Personality and Social Psychology, 97(4), 598-614. https://doi.org/10.1037/a0016683

Maio, G. R., Pakizeh, A., Cheung, W.-Y., \& Rees, K. J. (2009). Changing, priming, and acting on values: Effects via motivational relations in a circular model. Journal of Personality and Social Psychology, 97, 699-715.

McConahay, J. B., Hardee, B. B., \& Batts, V. (1981). Has racism declined in America? It depends on who is asking and what is asked. Journal of Conflict Resolution, 25(4), 563-579. https://doi.org/10.1177/002200278102500401

Merton, R. K. (1957). Social theory and social structure. New York: Free Press.

Montoya, R. M., Horton, R. S., \& Kirchner, J. (2008). Is actual similarity necessary for attraction? A meta-analysis of actual and perceived similarity. Journal of Social and Personal Relationships, 25(6), 889-922. https://doi.org/10.1177/0265407508096700

Murray, S. L., Holmes, J. G., Bellavia, G., Griffin, D. W., \& Dolderman, D. (2002). Kindred spirits? The benefits of egocentrism in close relationships. Journal of Personality and Social Psychology, 82(4), 563-581. 
Musiol, A.-L., \& Boehnke, K. (2013). Person-environment value congruence and satisfaction with life. International Journal of Humanities and Social Science, 3(9), 57-65.

Overall, N. C., Fletcher, G. J. O., Simpson, J. A., \& Fillo, J. (2015). Attachment insecurity, biased perceptions of romantic partners' negative emotions, and hostile relationship behavior. Journal of Personality and Social Psychology, 108(5), 730-749. https://doi.org/10.1037/a0038987

Overall, N. C., Clark, M. S., Fletcher, G. J. O., Peters, B. J., \& Chang, V. T. (2019). Does expressing emotions enhance perceptual accuracy of negative emotions during relationship interactions? Emotion. https://doi.org/10.1037/emo0000653

Overall, N. C., \& Hammond, M. D. (2013). Biased and accurate: Depressive symptoms and daily perceptions within intimate relationships. Personality and Social Psychology Bulletin, 39(5), 636-650. https://doi.org/10.1177/0146167213480188

Parsons, T., Shils, E. A., \& Olds, J. (1951). Values, motives, and systems of action. In T. Parsons \& E. Shils (Eds.), Toward a general theory of action: Theoretical foundations for the social sciences (pp. 47-275). New York: Harper.

Pettigrew, T. F., \& Tropp, L. R. (2006). A meta-analytic test of intergroup contact theory. Journal of Personality and Social Psychology, 90(5), 751. https://doi.org/10.1037/00223514.90.5.751

Richards, Z., \& Hewstone, M. (2001). Subtyping and subgrouping: Processes for the prevention and promotion of stereotype change. Personality and Social Psychology Review, 5(1), 52-73. https://doi.org/10.1207/S15327957PSPR0501_4

Roccas, S., Schwartz, S. H., \& Amit, A. (2010). Personal value priorities and national identification. Political Psychology, 31(3), 393-419. https://doi.org/10.1111/j.1467-9221.2010.00763.x Rokeach, M. (1973). The nature of human values. New York: Free Press.

Rokeach, M., \& Mezei, L. (1966). Race and Shared Belief as Factors in Social Choice. Science, 151(3707), 167-172. https://doi.org/10.1126/science.151.3707.167 
Rokeach, M., Smith, P. W., \& Evans, R. I. (1960). Two kinds of prejudice or one. The Open and Closed Mind, 132-168.

Sagiv, L., \& Schwartz, S. H. (2000). Value priorities and subjective well-being: Direct relations and congruity effects. European Journal of Social Psychology, 30(2), 177-198. https://doi.org/10.1002/(SICI)1099-0992(200003/04)30:2<177::AID-EJSP982>3.0.CO;2-Z

Sanderson, R., Prentice, M., Wolf, L., Weinstein, N., Kasser, T., \& Crompton, T. (2019). Strangers in a Strange Land: Relations Between Perceptions of Others' Values and Both Civic Engagement and Cultural Estrangement. Frontiers in psychology, 10, 559. doi:10.3389/fpsyg.2019.00559

Satchell, L. (2019). Discriminability in deception detection is not d: Reporting the Overlap Coefficient for practitioner-accessible results [Preprint]. https://doi.org/10.31234/osf.io/z4m2c

Schiefer, D., Möllering, A., \& Daniel, E. (2012). Cultural value fit of immigrant and minority adolescents: The role of acculturation orientations. International Journal of Intercultural Relations, 36(4), 486-497. https://doi.org/10.1016/j.ijintrel.2012.02.001

Schönbrodt, F. D., \& Humberg, S. (2018). RSA: An R package for response surface analysis (Version 0.9.12). Retrieved from https://cran.r-project.org/package=RSA

Schwartz, S. H. (1992). Universals in the content and structure of values: Theoretical advances and empirical tests in 20 countries. Advances in Experimental Social Psychology, 25, 1-65.

Schwartz, S. H., \& Bilsky, W. (1987). Toward a universal psychological structure of human values. 53(3), 550-562. https://doi.org/10.1037/0022-3514.53.3.550

Schwartz, S. H., \& Bilsky, W. (1990). Toward a theory of the universal content and structure of values: Extensions and cross-cultural replications. 58(5), 878-891. https://doi.org/10.1037/0022-3514.58.5.878 
Schwartz, S. H., Cieciuch, J., Vecchione, M., Davidov, E., Fischer, R., Beierlein, C., ... Konty, M. (2012). Refining the theory of basic individual values. Journal of Personality and Social Psychology, 103(4), 663-688. https://doi.org/10.1037/a0029393

Schwartz, S. H., Melech, G., Lehmann, A., Burgess, S., Harris, M., \& Owens, V. (2001). Extending the cross-cultural validity of the theory of basic human values with a different method of measurement. Journal of Cross-Cultural Psychology, 32(5), 519-542.

https://doi.org/10.1177/0022022101032005001

Solomon, S., \& Knafo-Noam, A. (2007). Value similarity in adolescent friendships. In T. C. Rhoades (Ed.), Focus on adolescent behavior research (pp. 133-155). New York: Nova Science.

Sortheix, F. M., \& Schwartz, S. H. (2017). Values that underlie and undermine well-being: Variability across countries. European Journal of Personality, 31(2), 187-201. https://doi.org/10.1002/per.2096

Stein, D. D., Hardyck, J. A., \& Smith, M. B. (1965). Race and belief: An open and shut case. Journal of Personality and Social Psychology, 1(4), 281-289. https://doi.org/10.1037/h0021870

Stephan, W. G., Ybarra, O., \& Bachman, G. (1999). Prejudice toward immigrants. Journal of Applied Social Psychology, 29(11), 2221-2237. https://doi.org/10.1111/j.1559$\underline{1816.1999 . t b 00107 . x}$

Stromberg, C., \& Boehnke, K. (2001). Person/society value congruence and well-being: The role of acculturation strategies. In P. Schmuck \& K. M. Sheldon (Eds.), Life goals and well-being (pp. 37-57). Hogrefe.

Tajfel, H., \& Turner, J. C. (1979). An integrative theory of intergroup conflict. In W. G. Austin \& S. Worchel (Eds.), The social psychology of intergroup relations (pp. 33-47). Monterey, CA: Brooks/Cole.

Thorndike, E. L. (1920). A constant error in psychological ratings. Journal of Applied Psychology, 
4(1), 25-29. https://doi.org/10.1037/h0071663

Turner, J. C., Hogg, M. A., Oakes, P. J., Reicher, S. D., \& Wetherell, M. S. (1987). Rediscovering the social group: A self-categorization theory. New York: Basil Blackwell.

Vandeselaere, N. (1991). The Impact of In-Group and Out-Group Homogeneity/Heterogeneity Upon Intergroup Relations. Basic and Applied Social Psychology, 12(3), 291-301. https://doi.org/10.1207/s15324834basp1203_4

Verplanken, B. (2004). Value congruence and job satisfaction among nurses: A human relations perspective. International Journal of Nursing Studies, 41(6), 599-605. https://doi.org/10.1016/j.ijnurstu.2003.12.011

Verplanken, B., \& Holland, R. W. (2002). Motivated decision making: Effects of activation and self-centrality of values on choices and behavior. Journal of Personality and Social Psychology, 82, 434-447.

Vecchione, M., Caprara, G., Schoen, H., Castro, J. L. G., \& Schwartz, S. H. (2012). The role of personal values and basic traits in perceptions of the consequences of immigration: A threenation study. British Journal of Psychology, 103(3), 359-377.

https://doi.org/10.1111/j.2044-8295.2011.02079.x

Vione, K. C. (2016). The effect of actual and inferred value similarity on interpersonal liking (PhD thesis, Cardiff University). Retrieved from http://orca.cf.ac.uk/96710/

Weidmann, R., Schönbrodt, F. D., Ledermann, T., \& Grob, A. (2017). Concurrent and longitudinal dyadic polynomial regression analyses of Big Five traits and relationship satisfaction: Does similarity matter? Journal of Research in Personality, 70, 6-15. https://doi.org/10.1016/j.jrp.2017.04.003

Wolf, L. J., Haddock, G., Manstead, A. S., \& Maio, G. R. (2020). The importance of (shared) human values for containing the COVID-19 pandemic. British Journal of Social Psychology, 59, 618-627. https://doi.org/10.1111/bjso.12401 
Wolf, L. J., Weinstein, N., \& Maio, G. R. (2019). Anti-immigrant prejudice: Understanding the roles of (perceived) values and value dissimilarity. Journal of Personality and Social Psychology, 117(5), 925-953. https://doi.org/10.1037/pspi0000177

Yoon, E., Chang, C.-T., Kim, S., Clawson, A., Cleary, S. E., Hansen, M., Bruner, J. P., Chan, T. K., \& Gomes, A. M. (2013). A meta-analysis of acculturation/enculturation and mental health. Journal of Counseling Psychology, 60(1), 15-30. https://doi.org/10.1037/a0030652

Zenker, S., Gollan, T., \& Quaquebeke, N. V. (2014). Using polynomial regression analysis and response surface methodology to make a stronger case for value congruence in place marketing. Psychology \& Marketing, 31(3), 184-202. https://doi.org/10.1002/mar.20686 